\title{
Methodische Probleme bei der radiographisch-densitometrischen Bestimmung der Dichte und der Jahrringbreiten von Holż
}

\author{
Von O. Lenz, E. Schär und F. H. Schweingruber
}

Eidgenössische Anstalt für das forstliche Versuchswesen, 8903 Birmensdorf, Schweiz

Schlüsselwörter

(Sachgebiete)

Methodik

Radiographie

Densitometrie

Digitale Datenerfassung

Jahrringbreite

Jahrringstruktur

Holzdichte

\author{
Methodische Probleme bei der radiographisch-densitometrischen Bestimmung \\ der Dichte und der Jahrringbreiten von Holz
}

\section{Zusammenfassung}

Das Hauptziel des weiteren Ausbaues der von Polge entwickelten Methode war es, die Holzdichte so genau wie möglich zu ermitteln und den Datenerfassungsvorgang zu automatisieren. Sowohl bei der Bohrkernentnahme wie beim Aufsägen der Proben in $3 \mathrm{~mm}, 2 \mathrm{~mm}$ und 1,25 $\mathrm{mm}$ dicke Späne wurde Wert auf größte Genauigkeit gelegt. Nach der Prüfung mehrerer Filmtypen und Entwicklungsverfahren verwenden wir einen RP-(rapid processing) Film, der bei gleicher Strahlendosis über die ganze Filmfläche hinweg gleiche Grauwerte aufweist. Mit der Radiographie von Celluloseacetatkeilen waren optisch nicht lineare Graukeile zu konstruieren, die auf jedern Film lineare Werte in Beziehung zur Holzdichte erzeugen. Technische Neuerungen am Mikrodensitometer (Joyce-Loebel) gestatten während der Dichteaufzeichnung eine Parallelisierung der Lichtmeßspalte mit den Jahrringgrenzen. Ein mit dem Mikrodensitometer gekoppeltes elektronisches Steuergerät ermöglicht die Übertragung der Daten auf einen externen Linienbandschreiber und zugleich auf ein Lochband. Kontinuierlich können die absoluten Dichtewerte alle 0,01 mm oder periodisch in Intervallen von $0,01-1 \mathrm{~mm}$ erfaßt werden. Die maximale und minimale Dichte und die Früh- und Spätholzbreiten auf einem vorgewählten Dichteniveau lassen sich selektiv auf das Lochband übertragen. Vergleichsmessungen haben gezeigt, daß in Hölzern mit schmalen Jahrringen nur unter Zuzug der Maximal- und Minimalwerte die reellen Kurven wiedergegeben werden. Mit dem am Steuergerät eingebauten Integrator ist die mittlere Dichte der Holzproben zu ermitteln. Die Differenz zwischen der reellen, mit klassischen Methoden errechneten Dichte und der radiographischen Dichte ergab, daß der Referenzkeil aus Celluloseacetat die Röntgenstrahlen stärker absorbiert als Holz. Es besteht somit die Möglichkeit auf radiographischem Wege die reelle Dichte des Holzes zu berechnen. Erste Analysen lassen vermuten, daß der RöntgenstrahlAbsorptionsgrad ein artspezifisches Merkmal ist.

Mots clés

Méthodologie

Radiographie

Densitométrie

Enregistrement numétique

Largeur du cerne

Structure du cerne

Densité du bois
Problèmes méthodologiques relatifs à la détermination de la densité et de la largeur des cernes annuels lors de l'analyse densitométrique de radiographies du bois

\section{Résumé}

Cette méthode, développée par Polge, a été améliorée dans le but de déterminer la densité du bois avec le plus d'exactitude et d'automatiser les mesures. Les perfectionnements portèrent également sur le prélèvement des éprouvettes de sondage et leur faconnage précis à des épaisseurs de 3,2 et $1,25 \mathrm{~mm}$. A la suite d'une série d'essais, nous utilisons un film permettant, par ses caractétéristiques, des mesures quantitatives et une machine automatique, assurant un développement homogène des radiographies. En plus, une corrélation linéaire rigoureuse pour l'échelle des densités optiques est établie pour chaque film au moyen de coins optiques non linéaires en acétate de cellulose. Des modifications furent apportées au microdensitomètre pour optimaliser l'exploration densitométrique, en particulier pour orienter le rayon lumineux parallèlement aux limites de cerne. Un équipement électronique, relié au microdensitomètre, a été construit pour la transcription automatique sur bande perforée des données des courbes densitométriques. Cet appareil nous donne la possibilité d'enregistrer les courbes de manière continue, soit tous les $0,01 \mathrm{~mm}$; de manière périodique, soit avec un intervalle régulier choisi de 0,01 à $1 \mathrm{~mm}$; et de manière sélective, en ne relevant que les maxima, les minima de densité, la largeur des cernes et la texture à un niveau de référence donné. Des mesures comparatives ont montré que, chez les bois à accroissements très fins, les intervalles d'enregistrement ne devaient pas dépasser $0,2 \mathrm{~mm}$ et que les valeurs extrèmes (maxima et minima) devaient être également notées pour conserver l'allure de la courbe. Enfin, la densité moyenne du bois peut être déterminée à partir de la radiographie à l'aide d'un intégrateur électronique incorporé à l'appareil. La différence constatée entre la densité réelle du bois, établie par la méthode classique, et la densité radiographique montre que le bois absorbe plus fortement les rayons $X$ que les coins de référence en acétate de cellulose. Il semble que le degré d'absorption soit spécifique à l'essence, la connaissance de celui-ci permet de corriger et de calculer la densité réelle du bois.

Methodological problems relative to the measurement of the density and width of growth rings by X-ray densitogrammes of wood

\section{Summary}

This method, which was developed by Polge, has been perfectioned in order to increase precision in the measurement of the density of wood and to permit the automation of the measuring process. The sampling cores were also improved and the internal form fashioned so as to obtain samples of accurate thicknesses of $3 \mathrm{~mm}, 2 \mathrm{~mm}$, and $1.25 \mathrm{~mm}$. After experimentation a film was selected whose characteristics permit quantitative measures. An automatic processor (Kodak RP, X-Omat, $\mathrm{M} 6 \mathrm{~A}-\mathrm{N}$ ) assures a homogenous processing of the radiographs. In addition, a linear correlation for the optical density scale was established for each film by means of a non-linear optical wedge
Methodology

Densitometry

Digital data acquisition

Growth ring width

Grow ring structure

Wood density 
of cellulose acetate. Several modifications of the microdensitometer were made to obtain an optimum densitometric scanning; in particular, the addition of mechanisms permitting the orientation of the light ray parallel to the boundary of the growth ring. A data-acquisition system connected to the microdensitometer was constructed to automatically transcribe on paper tape the information of the X-ray densitogrammes. This apparatus permits either a continuous registration of the curves every $0.01 \mathrm{~mm}$, or a periodic registration at regular intervals selected between 0.01 and $1 \mathrm{~mm}$, or a selective registration of the maximum and minimum densities, the width of growth rings, and the percentage of the late wood present at a given density level. Comparative measures reveal that for wood with narrow growth rings the registrative intervals should not be greater than $0.2 \mathrm{~mm}$ and that the peak values (maximum and minimum) should be plotted in order to conserve the trend of the curve. Finally, the mean density of wood can be evaluated from the radiogramme with the aid of an electric integrator incorporated in the apparatus.

A comparison between the density of wood as determined by the classic method (weight/volume) and the radiographic density shows generally higher values for the latter. It appears that the absorption of X-rays by wood is greater than that of the cellulose acetate step-wedges. The degree of absorption appears to be specific to the species of wood. With this knowledge the calculation of the density of wood by the radiographic method is feasible.

\section{Einleitung}

Dichte ${ }^{1}$ ), Jahrringbreite und Früh- und Spätholzanteile sind Kenngrößen des Holzes, die für die forstliche und technologische Holzforschung von größter Bedeutung sind. Die Dichte steht aber in engster Beziehung zu anatomischen, physikalischen, mechanischen und chemischen Eigenschaften des Holzes (Keller 1968). Die zur Zeit besten Dichte-Strukturdiagramme der Jahrringe sind mit Hilfe der radiographisch-densitometrischen Methode herzustellen.

Nachdem Lenz (1957) gezeigt hatte, daß sich Holz mittels Röntgenstrahlen in allen Einzelheiten abbilden läßt, hat Polge (1963 und 1966) die radiographisch-densitometrische Methode zur Dichteanalyse des Holzes entwickelt. Seither hat er die Methode weiter ausgebaut, mehrere Wissenschafter haben seine Gedanken übernommen und neue Apparate hergestellt und beschrieben (z. B. Mc Neely et al. 1973, Parker I97I und 1973, Polge 1971 a). Basierend auf diesen Erkenntnissen wurde 1973 bis 1975 an der Eidgenössischen Anstalt für das forstliche Versuchswesen (EAFV), Birmensdorf, eine Apparatur aufgebaut, mit dem Ziele, die Erforschung der Holzqualität und der Beziehungen des Holzes zum Standort zu vertiefen, wobei die von Polge begründete Methode soweit als möglich verbessert und die Datenerfassung rationalisiert werden sollte.

Wir stellen vorerst schematisch und summarisch den Arbeitsablauf, die vorhandenen Fehlerquellen und deren Eliminierung vor ( $T a b .1$ ) und beschreiben anschließend die an der EAFV erarbeiteten technischen Verbesserungen.

\section{Präparation und Bestrahlung des Materials}

\section{I Probenentnahme}

Das von uns angewendete Bohrverfahren mit dem Zuwachsbohrer ist insbesondere von Polge (1966; I971 b) und Reineke (I94I) beschrieben worden.

Zur Erfassung der Dichte ist die genaue Orientierung und Lokalisierung der Proben im Baum eine Notwendigkeit (Arbeitsgruppe 1966). Aus diesem Grunde wurde eine Bohr-

1) Unter Dichte verstehen wir die Rohdichte (nach DIN 52 182), gemessen bei bestimmter Holzfeuchtigkeit, in unserem Falle bei $8 \%$.

Dichte $=r_{B}=\frac{G_{B}}{\text { (Gewicht der Probe bei } 8 \% \text { Feuchtigkeit) }}$ $\mathrm{g} / \mathrm{cm}^{3}=r_{8}=\frac{G_{8}}{V_{8}}$ (Volumen der Probe bei $8 \%$ Feuchtigkeit) Bei der Bestrahlung sind die Proben auf einen Wassergehalt von $8 \%$ konditioniert.

Synonyme: Rohwichte, Raumgewicht, franz.: Densité à $8 \%$ d'humidité, engl.: Density at $8 \%$ moisture content.

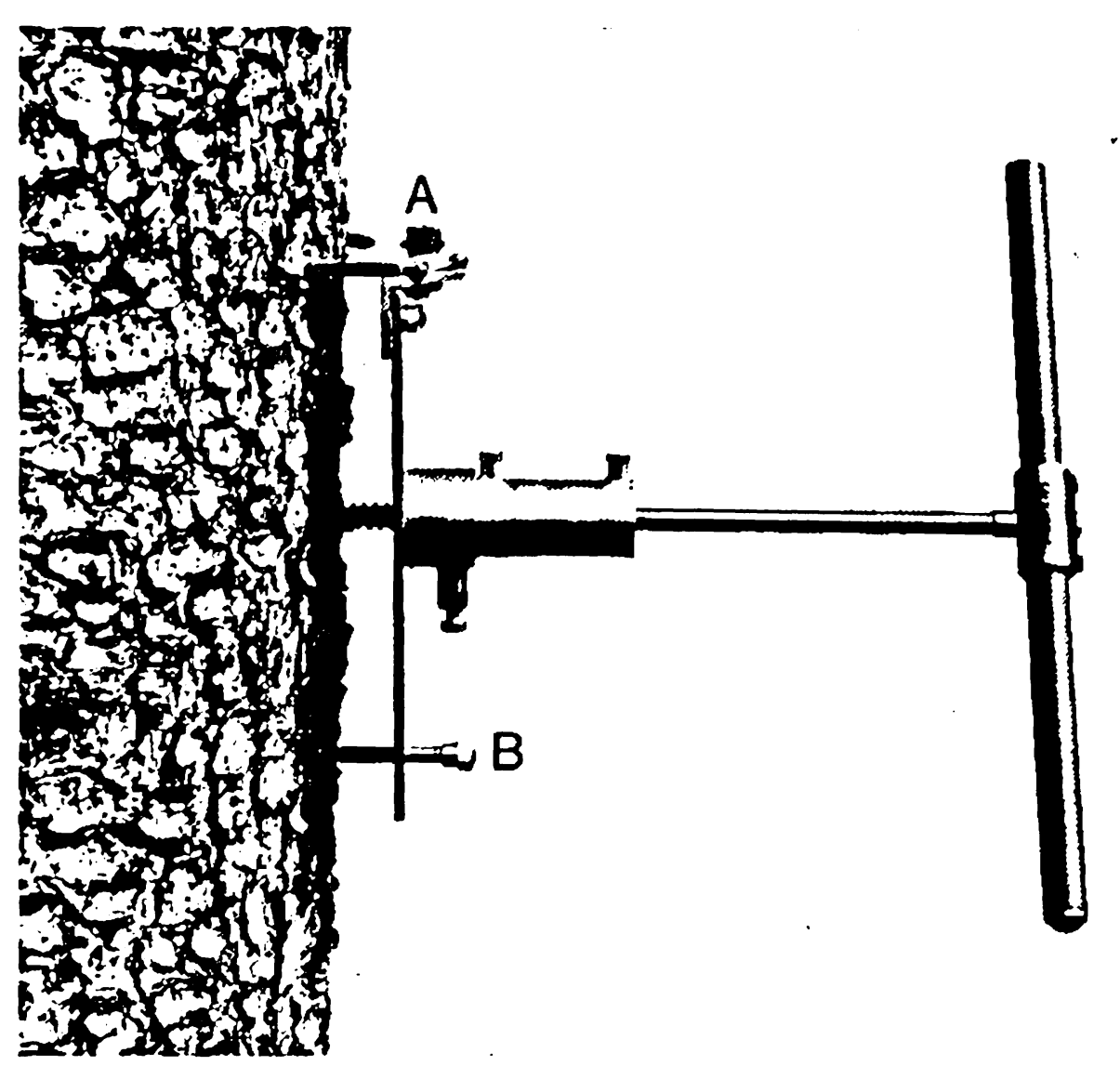

Abb. I. Halterung und Führung des Zuwachsbohrers zur Bestimmung der Bohrrichtung. Das Gelenk bei Schraube A erlaubt eine radiale Orientierung des Bohrkerns. Mit der Schraube B kann der Bohrer senkrecht zur Stammachse ausgerichtet werden

The guide for the orientation of the increment bore allows a precise selection of the drill line. The articulation (A) permits the radial orientation of the bore. With the screw (B) the bore can be adjusted perpendicular to the axis of the trunk

orientierungshilfe (Abbildung I) gebaut, die es erlaubt, Bohrkerne senkrecht zur Stammachse oder zur Stammoberfläche zu entnehmen ${ }^{2}$ ). Sind Bohrkerne trotz dieser Einrichtung nicht ganz genau orientiert, ist eine Nachorientierung in beschränktem Maße, bei der Herstellung der parallelflächigen Proben, mittels einer beweglichen Schnellspannvorrichtung möglich (Abb. 4). Die durch die Strahlengeometrie der Röntgenquelle und der doppelseitigen Filmbeschichtung unvermeidbaren Verzerrungen der Jahrringbilder auf dem Film spielen, im Vergleich zur mangelhaften Orientierung der Proben im Stamm, eine untergeordnete Rolle (Polge 1966; 1969). Maßgebend für die densitometrische Auswertung ist die Schärfe der Röntgenbilder.

Aus Abbildung 2 geht hervor, daß die Faserneigung die Resultate der Extremwerterfassung, insbesondere die maximale Dichte in Jahrringen mit schmalem Spätholzanteil, sehr stark beeinflußt. Es ist vor allem bei Arbeiten, bei denen die von Jahr zu Jahr unterschiedlichen maximalen Dichteniveaus mit irgendwelchen anderen Parametern (Klimadaten, pedologische Daten, forstliche Maßnahmen u. a.) in Beziehung gebracht werden, von größter Wichtigkeit, daß die Jahrringe genau senkrecht zur Stammachse orientiert sind. Selbst kleinste Ab-

2) Um sekundäre Schädigungen des Holzes durch Pilze zu vermeiden, werden die Bohrlöcher mit einer Wundverschlußpaste ausgefüllt: Lack-Balsam unter Zugabe von $4 \% \mathrm{CB}$ Wolmanit $(\mathrm{Cu}, \mathrm{K}, \mathrm{B})$. (Lenz und Oswald 1971; Polge und Thiercelin 1970; Thiercelin et al. 1972). 
Tabelle I

Arbeitsablauf und Methodik bei der radiographisch-densitometrischen Erfassung der Holzdichte Working process and methodology of radiographic-densitometric measurement of the wood density

\begin{tabular}{l|l|l}
\hline Arbeitsschritte & Wichtigste Mängel oder Fehlerquellen & $\begin{array}{l}\text { Behebung der Mängel und Reduzierung der Fehler- } \\
\text { quellen durch Arbeitshilfen }\end{array}$ \\
\hline $\begin{array}{l}\text { Bohrspanentnahme } \\
\text { Bohren mit Zuwachsbohrer }\end{array}$ & $\begin{array}{l}\text { Schlechte Orientierung der Bohrkerne } \\
\text { in vertikaler Richtung }\end{array}$ & Konstruktion einer Bohrerführung
\end{tabular}

Transport der Bohrkerne

Schutz des Baumes vor Pilzinfektion

Zwischenlagerung

\section{Harzextraktion}

Herstellung der Proben Verarbeitung der zylindrischen Bohrkerne zu parallelflächigen Bohrspänen

Beschriftung der Bohrspäne

Herstellung
der Radiogramme
Belegung der Filme mit Proben

Filmwahl

Bestrahlung

Filmentwicklung

\section{Densitometrieren}

Linearisierung der optischen Dichte mit der Holzdichte

Koordinierung der Lichtmeßspalte mit dem Jahrringverlauf

Vergleich der Absorptionseigenschaften von Celluloseacetat und Holz

Datenerfassung

Analogdaten

Digitale Daten
Schlechte Orientierung der Bohrkerne
in vertikaler Richtung

Bau von leichten, wasserdurchlässigen Bohrkernetuis Einbringung einer hydrophilen, fungiziden Paste ins Bohrloch

Harze trocknen ein und beeinflussen die Dickengenauigkeit bei Probenherstellung

Harze sind mobile Exkrete und bilden nicht Bestandteil einzelner Jahresringe

Runde Bohrkerne eignen sich schlecht zur densitometrischen Auswertung, da die Dicken von o- $5 \mathrm{~mm}$ variieren

Lagerung der Proben in 80\% igem Alkohol

Extraktion der Harze in 96\%igem Alkohol oder Aceton im Soxhlet

Die runden Bohrkerne werden zu Spänen von $3 \mathrm{~mm}$, $2 \mathrm{~mm}$ oder I, $25 \mathrm{~mm}$ Dicke verarbeitet:

- Bau einer Verleimungseinrichtung und einer Schnellspannvorrichtung

- Aufsägen der runden Bohrkerne zu parallelflächigen Bohrspänen mit einer Präzisions-Doppelkreissäge

Die Proben werden mit Tusche auf der Stirnseite der Späne beschriftet

Filme werden bei unsachgemäßer $\mathrm{Be}-$ handlung verletzt und ergeben unerwünschte Schwärzungen

Nicht alle im Handel erhältlichen Filme eignen sich zur quantitativen Analyse

Diverse. Siehe Polge 1966

Nicht homogene Entwicklung, resp. Schwärzung des Films über die ganze Fläche

Jahrringe verlaufen nicht parallel zu der längeren Achse des Lichtmeßspaltes

Celluloseacetat und $\mathrm{Holz}$ absorbieren Röntgenstrahlen nicht in gleichem Maße

Die maximale Vorschubstrecke des Schreibers am Mikrodensitometer ist für unsere Bedürfnisse zu gering

Der Arbeitsaufwand ist bei der manuellen Datenentnahme von der Kurve sehr hoch

\section{Herstellung eines Probenträgers}

Wahl von gleichmäßig beschichteten, feinkörnigen Filmtypen

Bestrahlung der Proben aus 2,5 m Distanz. Ermittlung der optimalen Dosis

Ermittlung der optimalen Bestrahlungsfläche

Die Bestrahlungskabine muß allen Vorschriften hinsichtlich Strahlenschutz genügen

Entwicklung der Filme im Kurzzeit-Entwicklungsautomat

Herstellung eines Stufenkeils und eines stufenlosen Keils

Bau einer simultanen Steuerung des Kompensationsprismas am Mikrodensitometer mittels Servomotor und der Lichtmeßspalte

Vergleich der gravimetrisch-volumetrischen mit der radiographischen Dichtebestimmung von Celluloseacetat und Holz

Anschluß eines externen Linienschreibers

Bau eines Datenerfassungs- und Steuergerätes zu kontinuierlicher, periodischer und selektiver Registrierung von Daten weichungen, die auf den Filmen bei genauer Betrachtung als Unschärfen (flou) erkannt werden können, sind für die Auswertung nicht tolerierbar. Bei $3 \mathrm{~mm}$ dicken Bohrspänen sind die durch die schräge Faserstellung verursachten Fehler wesentlich größer, als bei Spänen mit einer Dicke von $1,25 \mathrm{~mm}$.

Keine integralen Dichteunterschiede ergeben sich zwischen senkrecht und schief orientierten Proben.

Oft sind die Jahrringe auf dem Querschnitt der Bohrspäne nicht genau senkrecht orientiert, sei es infolge Wuchseigenschaften des Baumes oder nicht zentrischem Anbohren. Die hiermit durch den $\mathrm{Mi}$ krodensitometer falsch wiedergegebenen Dichtewerte konnten durch den Einbau eines Servomotors am Kompensationsprisma korrigiert werden. Somit können nun die Jahrringgrenzen mit der spaltförmigen Leuchtfeldblende (Lichtmeßspalte), simultan parallel, orientiert werden. Abbildung 3 zeigt, daß in Hölzern mit sehr schmalen Spätholzzonen, Abweichungen der Jahrringgrenzen von der Senkrechten, bzw. von der Lichtmeßspalte, kleiner als $2,5^{\circ}$ sein müssen, sollen nicht die Ergebnisse in zu starkem Maße verfälscht werden. Eine Abweichung der Jahrringgrenzen von der Lichtmeßspalte um $10^{\circ}$ entspricht in ihren maximalen Dichtewerten ungefähr einer Faserneigung von $2^{\circ}$ bei $3 \mathrm{~mm}$ dicken Proben. Abweichungen der Jahrringgrenzenstellung von $5^{\circ}$ entsprechen; einer Faserneigung von $6^{\circ}$ in $1,25 \mathrm{~mm}$ dicken Proben.' 

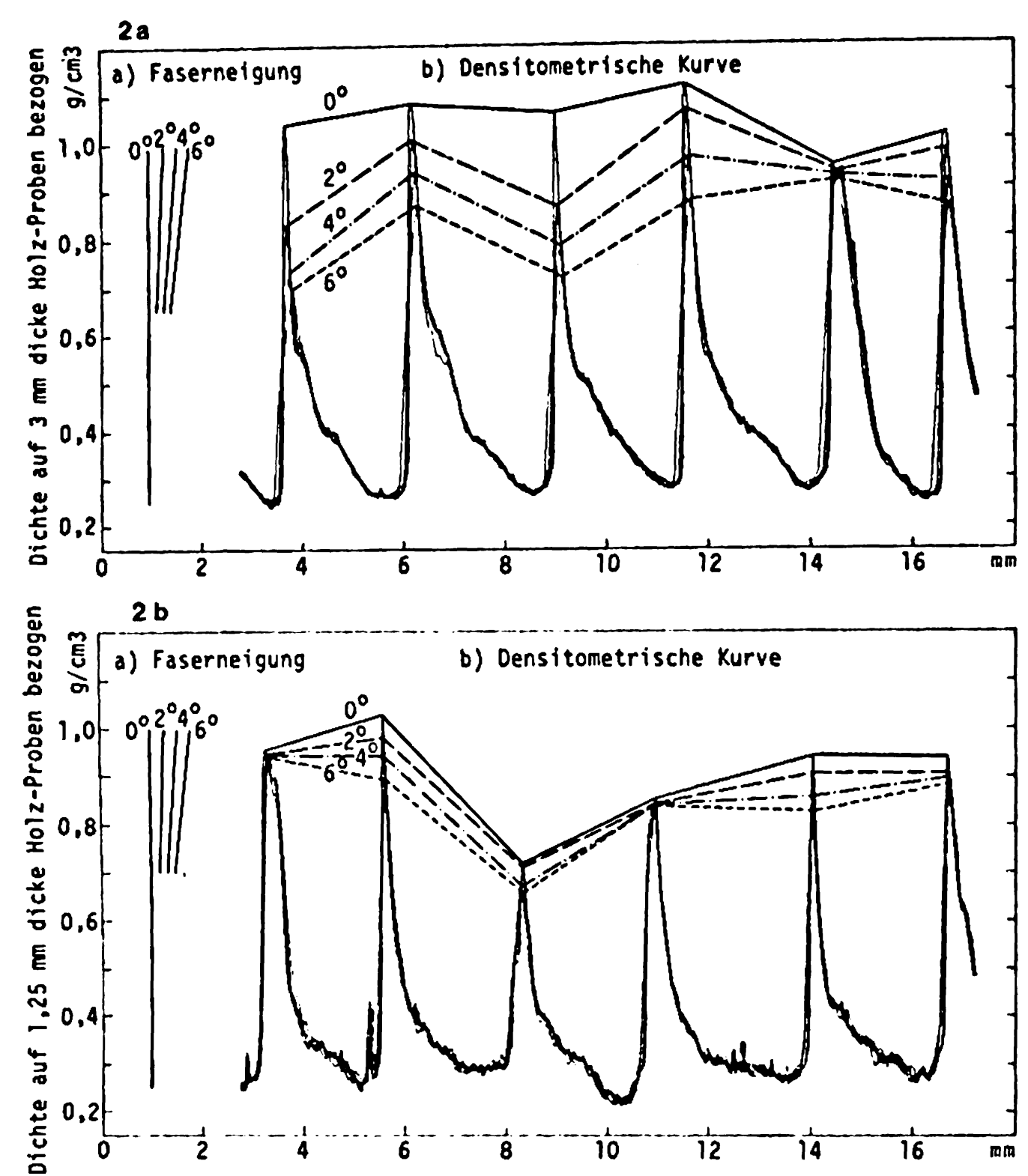

Abb. 2. Einfluß der Faserneigung und der Probendicke auf die radiographische Abbildung und deren Auswirkung auf die

Extremwerterfassung (maximale Dichte) im Jahrring

a) $3 \mathrm{~mm}$ dicke Probe von Fichte

b) I,25 mm dicke Probe von Fichte

The influence of fiber direction and sample-thickness on the radiographic image and the maximum density of growth rings

a) a spruce sample with a thickness of $3 \mathrm{~mm}$

b) a spruce sample with a thickness of $1.25 \mathrm{~mm}$

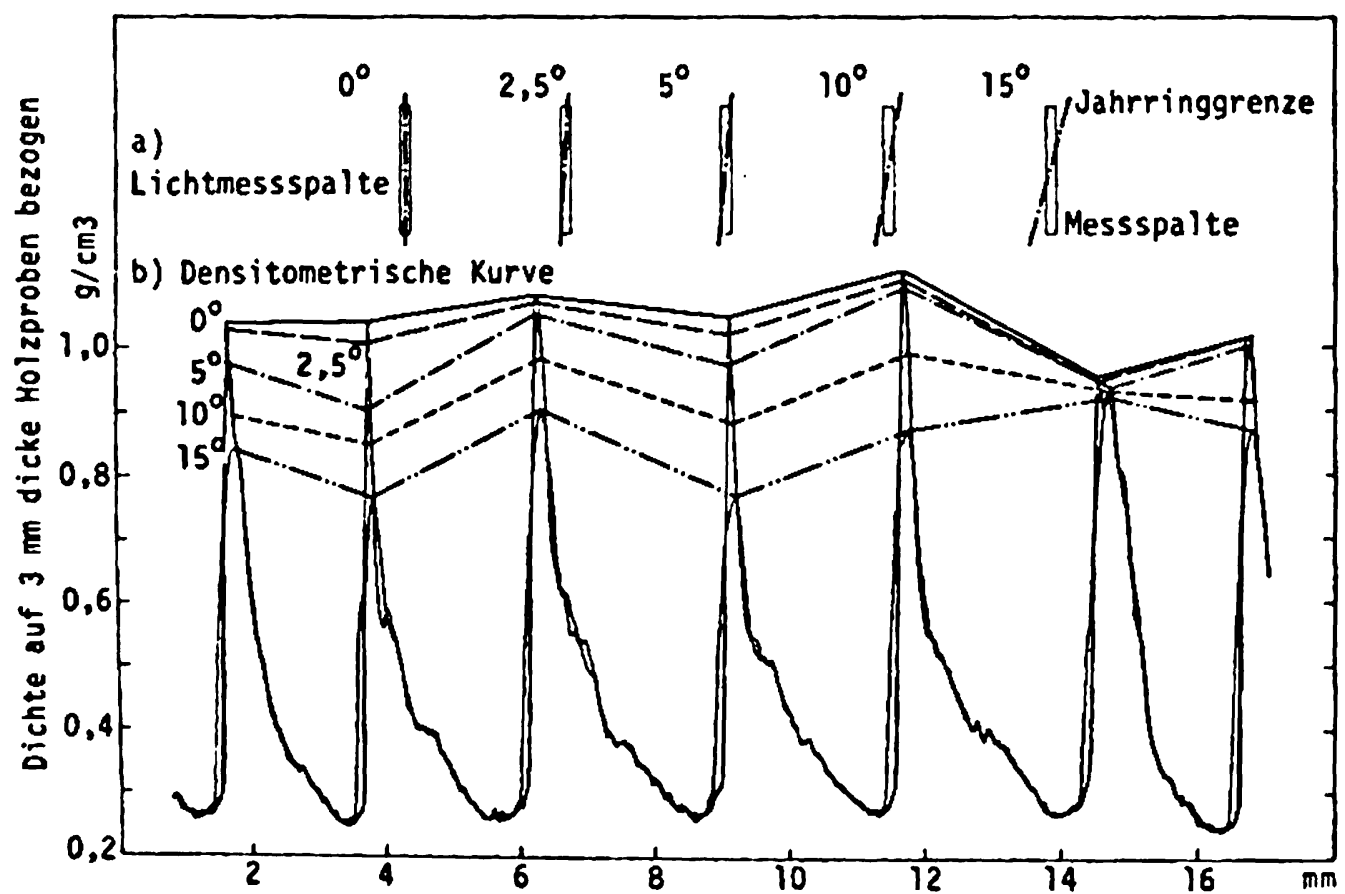

Abb. 3. Einfluß der schiefen Jahrringstellung auf die Extremwerterfassung (maximale Dichte). Erst Abweichungen von mehr als $2,5^{\circ}$ verfälschen die Resultate wesentlich

The influence of an oblique position of the growth boundary on the maximum density. Any deviation greater than $2.5^{\circ}$ considerably falsifies the results

\subsection{Herstellung der Bohrspäne}

$\mathrm{Da}$ die optische Dichte auf dem Film direkt abhängig ist von der Probendicke, werden die Bohrkerne oder die Späne aus Stammscheiben mit einer Doppelkreissäge (Zwillingssäge) $)^{3}$ ) zu parallelflächigen Bohr-

\footnotetext{
3) Sägeblätter: Hohlgeschliffene Voll-Hartmetallkreissägen $63 \times 1 \times 16$, 100 Zähne der Firma Usines métallurgiques de Vallorbe $\mathrm{CH}-\mathrm{r} 337$ Vallorbe. Präzisions-Bohr- und Fräsmaschine P I8 S der Firma Fehlmann, $\mathrm{CH}-5703$ Seon.
}

16 Holzforschung Bd. 30, Heft 4 spänen verarbeitet [(Kuszec 1972; Polge und Nicholls 1972; Thiercelin und Perrin 1972) Abb. 4]. Bei fachgerechter Bedienung der Maschine werden routinemäßig Proben mit einer Toleranz von $0,01-0,02 \mathrm{~mm}$ Dicke hergestellt. Die normale Spandicke beträgt $3 \mathrm{~mm}$, sind die Jahrringe jedoch sehr schmal oder schief zur Stammachse orientiert, 2 oder I,25 mm. Bei der Auswertung werden die Dickeabweichungen berücksichtigt.

\subsection{Bestrahlung und Entwicklung der Filme}

Bei der quantitativen densitometrischen Analyse ist insbesondere der homogenen Schwärzung auf der ganzen Filmfläche und der Orientierung des Jahrrings zum Meßsystem Beachtung zu schenken.

Die Schwärzung der Filme ist abhängig von der gleichmäßigen Bestrahlung, der Filmqualität und der Entwicklung.

a) Orientierung der Röntgenquelle ${ }^{4}$ )

Die Orientierung der Röntgenquelle, bzw. die Bestimmung der Fläche mit gleicher Strahlendosis, erfolgte vorerst mittels Geiger-Zähler und abschließend mit der densitometrischen Prüfung von verschiedenen, im Strahlungsfeld ausgelegten Filmen ( $12 \mathrm{kV}, 20 \mathrm{~mA}$, $80 \mathrm{~min}$ ). Die optimal nutzbare, lotrecht 2,5 m unterhalb der Röntgenquelle liegende Kreisfläche besitzt einen Durchmesser von mindestens $30 \mathrm{~cm}$, wobei die

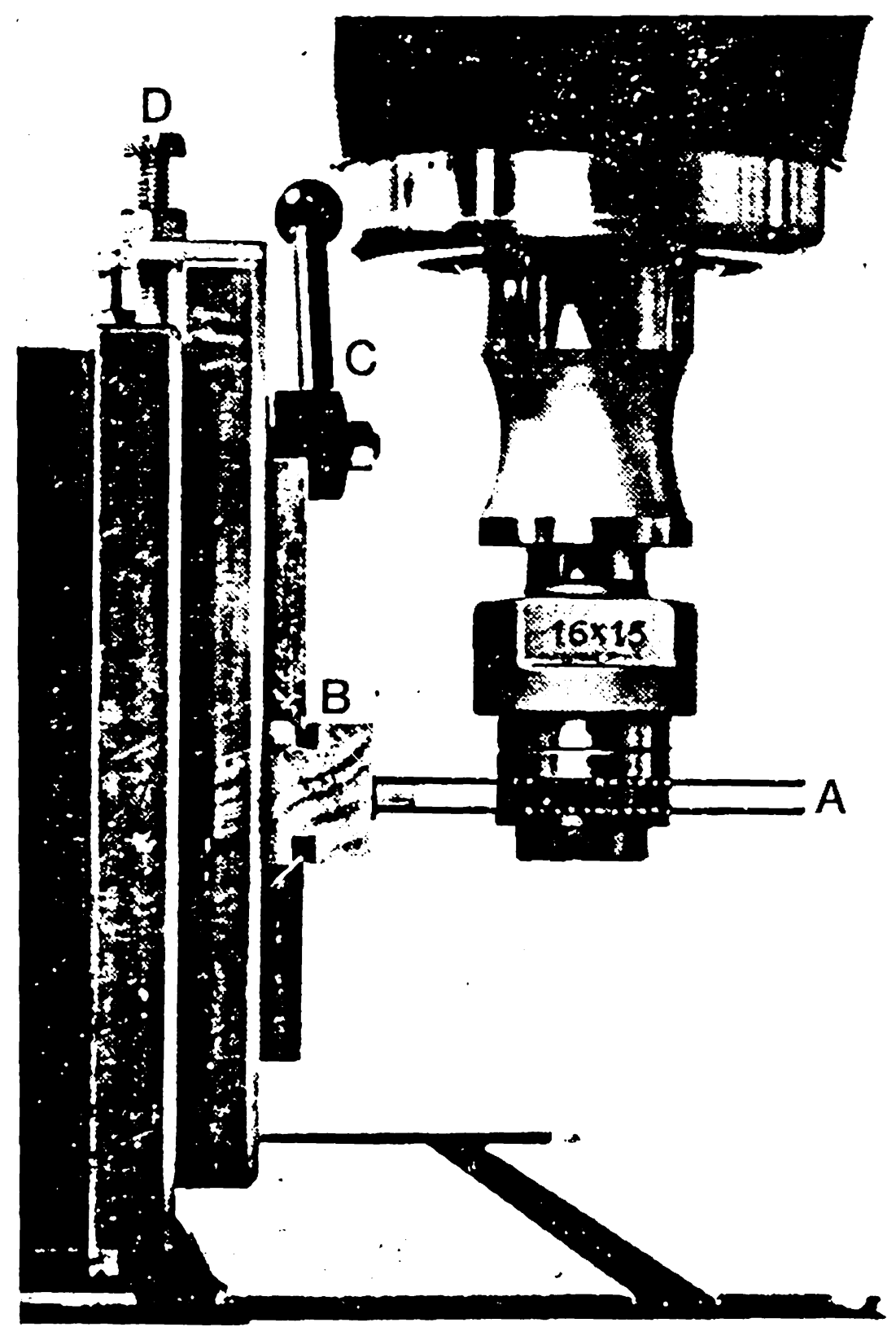

Abb. 4. Doppelkreissäge aus Hartmetallblättern (A) an einer Metallbearbeitungsmaschine. Die Proben werden auf eine Holzleiste aufgeleimt (B), die mit einer Schnellspannvorrichtung (C) auf einer Metallplatte festgeklemmt wird. Eine beschränkte

Nachorientierung ist mittels Stellschraube (D) möglich

A carbide twin blade (A) on a metalworking machine. The samples are glued to a wooden strip (B) and fixed with a clamping device (C) to a metal plate. Minute adjustments of the orientation are possible by means of screw (D)

$\left.{ }^{4}\right)$ Generator: Baltcau, Baltographe 5-50 kV, Typ BF 50/20. Röntgenröhre: Tubix, Typ GLA 25, W 5. 
Streuung der optischen Dichte einer Variation von $0,007 \mathrm{~g} / \mathrm{cm}^{3}$ Holzdichte, bei $3 \mathrm{~mm}$ dicken Proben, entspricht.

b) Gleichmäßig beschichtete, nicht durch Wärme oder Druck lokal aktivierte, feinkörnige Filme liefern für unsere Zwecke die besten Ergebnisse (Abb. 5). Aufgrund dieser Versuche verwenden wir den Film Kodak, RP/M.

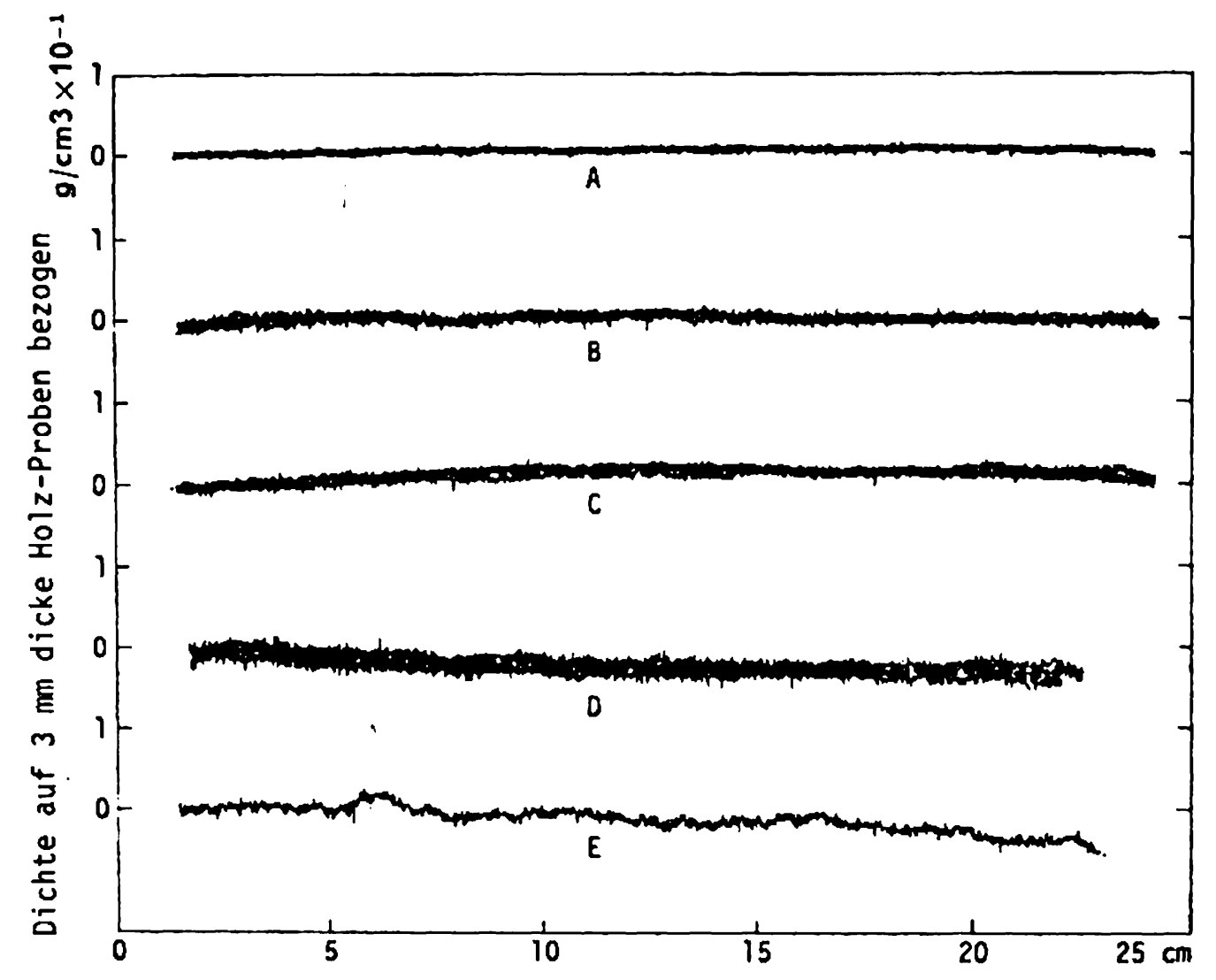

Abb. 5. Dichtestreuung verschiedener Filmprodukte:

\begin{tabular}{|c|c|c|c|c|}
\hline Produkt & Beschichtung & $\begin{array}{c}\text { Span- } \\
\text { nung }\end{array}$ & Zeit & $\begin{array}{l}\text { Streuung } \\
\text { der Dichte } \\
\text { um den } \\
\text { Mittelwert } \\
\text { in } \mathrm{g} / \mathrm{cm}^{3}\end{array}$ \\
\hline $\begin{aligned} \mathrm{A}= & \text { Kodak, } \mathrm{RP} / \mathrm{M} \\
\mathrm{B}= & 3 \mathrm{M} \text { Medical } \\
& \text { X-Ray. Typ S }\end{aligned}$ & $\begin{array}{l}\text { beidseitig } \\
\text { beidseitig }\end{array}$ & $\begin{array}{l}16 \mathrm{kV} \\
14 \mathrm{kV}\end{array}$ & 10 $\mathrm{min}$ & $\begin{array}{l}0,007 \\
0,012\end{array}$ \\
\hline $\begin{aligned} \mathrm{C}= & \text { Kodak PE } 4006 \\
\mathrm{D}= & \text { Agfa CURIX } \\
& \text { RPl-PE-FW }\end{aligned}$ & $\begin{array}{l}\text { beidseitig } \\
\text { beidseitig }\end{array}$ & $\left|\begin{array}{l}14 \mathrm{kV} \\
13 \mathrm{kV}\end{array}\right|$ & $\begin{array}{r}4 \mathrm{~min} \\
\text { 10 } \mathrm{min}\end{array}$ & $\begin{array}{l}0,017 \\
0,030\end{array}$ \\
\hline$E=$ Kodak RP/SU & einseitig & $|19 \mathrm{kV}|$ & $8 \mathrm{~min}$ & 0,035 \\
\hline
\end{tabular}

Distanz Film - Röntgenquelle 2,5 m. Stromstärke $20 \mathrm{~mA}$

Mit Ausnahme von Film E wurden 3 Kurven aus dem oberen, mittleren und unteren Teil des Films übereinandergelegt (Eichung auf $3 \mathrm{~mm}$ dicke Holzproben). Das eindeutig beste Ergebnis lieferte Film A mit einer Streuung von $0,007 \mathrm{~g} / \mathrm{cm}^{3}$ um den Mittelwert

Density scatter of various film products:

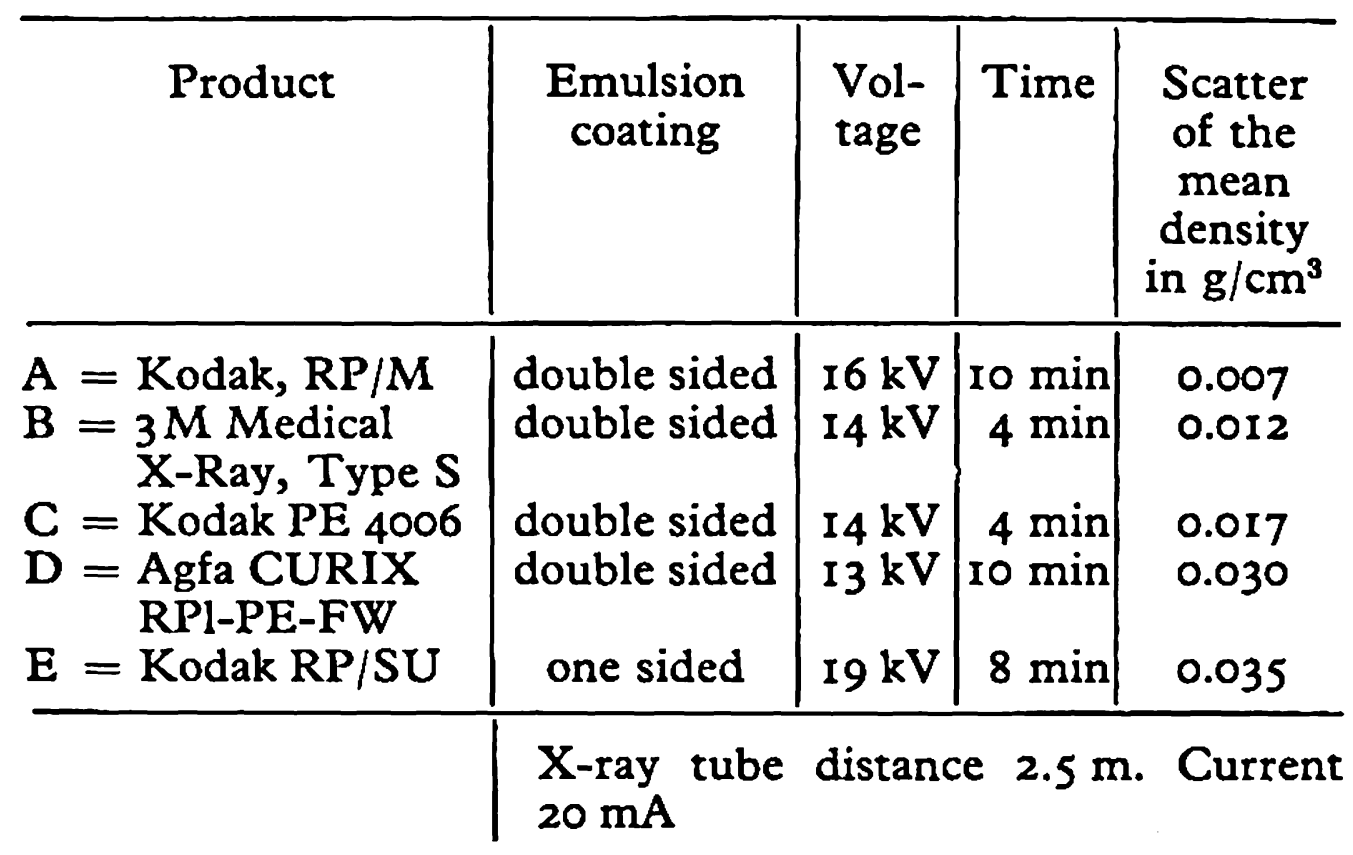

Three curves, calibrated for $3 \mathrm{~mm}$ thick samples, were taken in the upper, middle and lower sections of each film (with the exception of film E) and superimposed. Unequivocally the best results were obtained with film $A$ which has an average scattering of $0.007 \mathrm{~g} / \mathrm{cm}^{3}$
Zur Vermeidung aller sekundären Filmbeschädigungen (Staub, Druckbelastung, Knickung, Belichtung des Films durch Dunkelkammerbeleuchtung ${ }^{5}$ ) wurde eine Halterung mit einer Zellophanfolie gebaut, die es erlaubt, eine größere Anzahl Bohrspäne, gegenüber dem Eichteil und nach der Nummer genau orientiert, bei Tageslicht in aller Ruhe zu plazieren (Abb.6). Erst unmittelbar vor der Bestrahlung wird der Film aus der Schutzhülle genommen und unter die vorbereitete Probenserie gelegt. Die Proben werden mit Tusche auf der Schnittfläche beschriftet, da die densitometrische Prüfung beschrifteter und unbeschrifteter I $\mathrm{mm}$ dicker Holzproben und $0,1 \mathrm{~mm}$ dicker Papierproben keine meßbaren Unterschiede ergeben hat.

Anstelle der in der Literatur empfohlenen Entwicklungsverfahren (Parker und Meleskie 1970; Polge und Nicholls 1972) wird mit einem Röntgenfilm-Entwicklungsautomaten (90-sec-Zyklus) ${ }^{6}$ ) und den diesem Verfahren angepaßten, feinkörnigen Filmen, gearbeitet. Diese Methode liefert uns, bei serienmäßigen Arbeiten, durchschnittlich bessere Resultate als die Langzeitverfahren. Die durch die Röntgenabteilung des städtischen Krankenhauses praktisch voll ausgelasteten Geräte garantieren eine regelmäßige und fachgerechte Wartung und somit auch stets gleichartig entwickelte Filme. Die densitometrische Prüfung der Filme ergab, daß mit Ausnahme eines ungefähr $2 \mathrm{~cm}$ breiten Streifens und selten auftretender schwarzer Punkte (Walzenverunreinigung in den Entwicklungsautomaten), die Filme auf der ganzen Fläche gleichmäßig entwickelt sind (Abb. 5).

\section{Messung der Holzdichte}

Zur Ermittlung der Holzdichte wird in Anlehnung an Polge (1966; 1969), Fletcher und Hughes (1970), Polge und Nicholls (1972), Parker und Jocza (1973) ein Eichkeil aus Celluloseacetat mit Dickenstufen und einem kontinuierlich zunehmenden Dickenkeil (Abb. 7a) mit den zu analysierenden Hölzern mitbestrahlt. Dabei

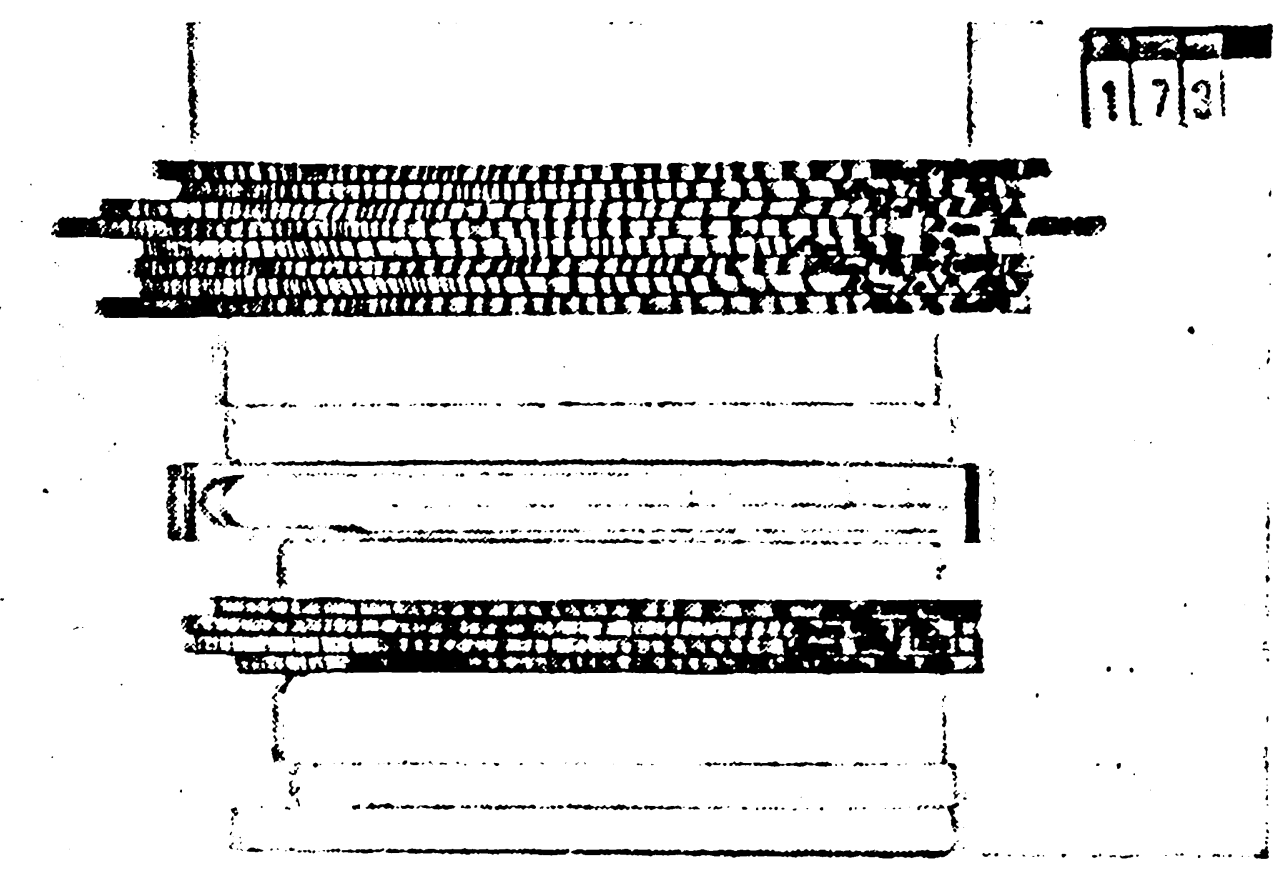

Abb. 6. Probenhalterung mit Cellophanfolie. Jeder Film wird mit Holzproben (Beschriftung mit Tusche auf Stirnseite), einem Referenzkeil aus Celluloseacetat und einer Nummer mit bleihaltiger Farbe belegt. Nach Möglichkeit bleiben allseits je $2 \mathrm{~cm}$ Filmfläche frei

Sample tray with a cellophane sheet bottom. The wood samples (inscribed with Indian ink on the front face), a celloluse acetate step-wedge, and X-ray-opaque reference marker are placed on each film. A $2 \mathrm{~cm}$ border is left around each film

5) Dunkelkammerbeleuchtung: Natrium-Dampfröhre Diffuseur Duplex.

$\left.{ }^{6}\right)$ Entwicklungsautomat: Kodak RP, $\dot{\mathrm{X}}$-Omat M6 A-N. 


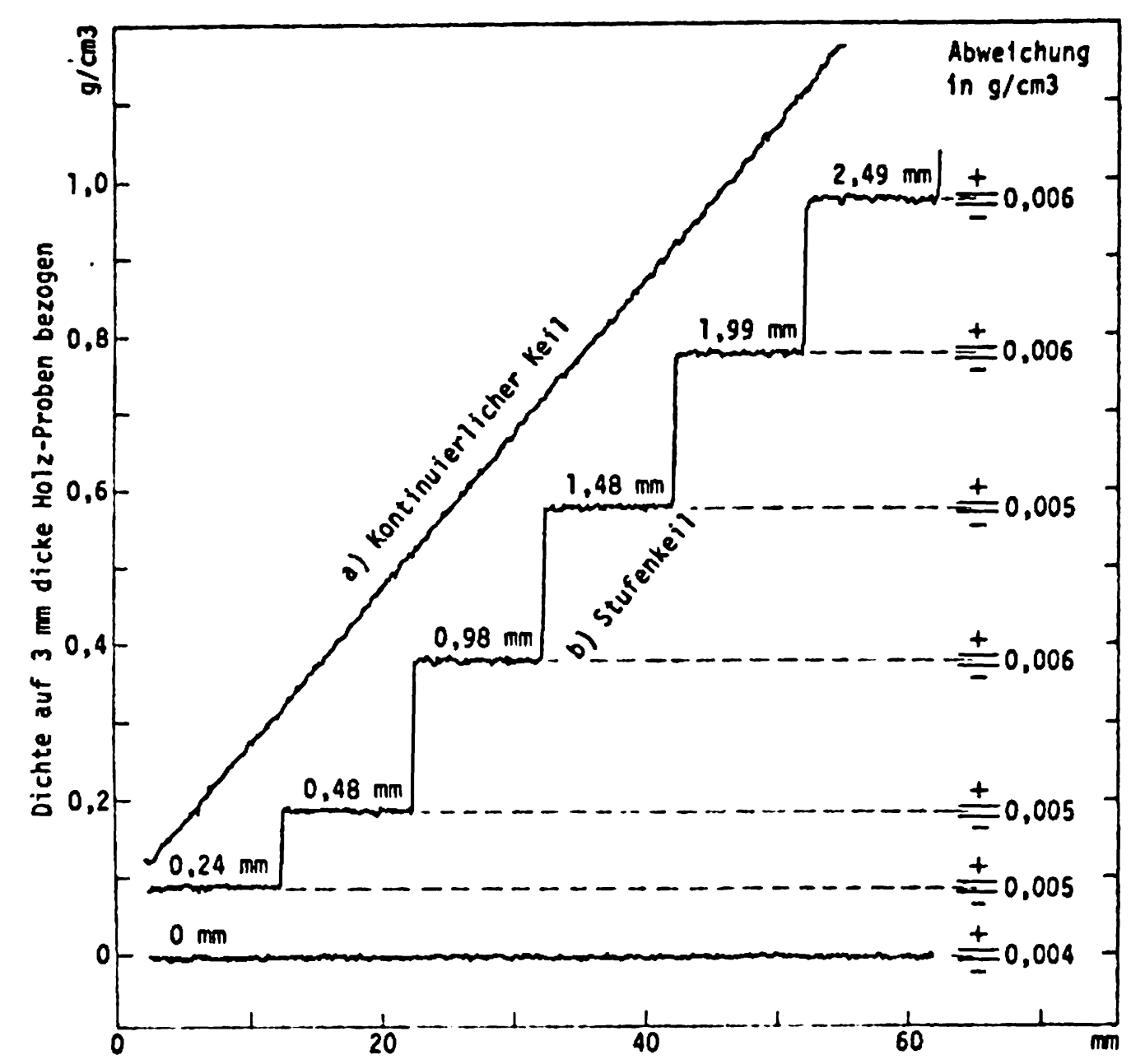

a) Kontinuierlicher Keil

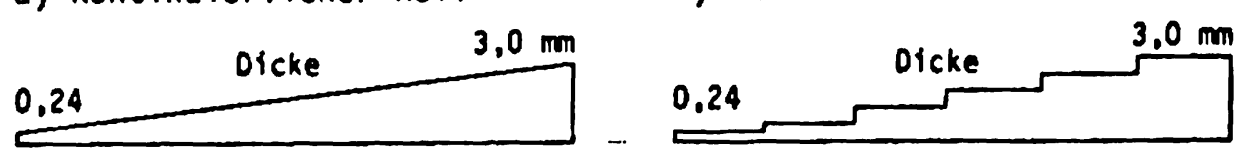

Abb. 7. Radiogramm eines Celluloseacetat-Keils. Bestrahlung: I $7 \mathrm{kV}, 20 \mathrm{~mA}, 6 \mathrm{~min}$. Eichung auf $3 \mathrm{~mm}$ Holz. Die Abweichung vom Mittelwert beträgt maximal $\pm 0,006 \mathrm{~g} / \mathrm{cm}^{3}$

Bei Verwendung eines optisch nicht linearen Keils im Referenzstrahl des Mikrodensitometers werden holzdichtemäßig lineare Werte erzielt.

Radiograph of cellulose acetate step-wedge. Ray-treatment: $17 \mathrm{kV}, 20 \mathrm{~mA}, 6 \mathrm{~min}$. calibrated for $3 \mathrm{~mm}$ thick wood samples. The maximum deviation from the mean density is \pm 0.006 $\mathrm{g} / \mathrm{cm}^{3}$.

By exposing the optically non-linear wedge to the reference ray of the microdensitometer, a linear value for the wood's density is obtained

wird vorerst angenommen, daß Holz und Celluloseacetat (Dichte 1,27$)^{7}$ ) annähernd die gleichen $\mathrm{Ab}$ sorptionseigenschaften für Röntgenstrahlen aufweisen. Nach der Bestrahlung werden die Filme in einem Doppelstrahl-Micro-Densitometer (Joyce-Loebl MK III CS) analysiert ${ }^{8}$ ), wobei die Grauwerte des Eichkeils mit denen des Holzes verglichen werden. Durch den im Referenzstrahl eingebauten, optisch linearen Graukeil ergibt sich keine Linearität in Beziehung zur Dichte des Celluloseacetats, resp. des Holzes. Deshalb wird der optisch lineare durch einen optisch nicht linearen Graukeil (Radiographie eines Celluloseacetatkeils) ersetzt, der aber in Beziehung zur Celluloseacetatdichte lineare Werte erzeugt. In Verbindung mit leichten Verschiebungen im Filter-Graukeil des Referenzstrahls erzeugt der Mikrodensitometer holzdichtemäßig lineare Werte (Abb. 7). Die Holzdichte in $\mathrm{g} / \mathrm{cm}^{3}$ ist ermittelbar:

Dichte des Celluloseacetats $\times$ Dicke der Celluloseacetatstufen Dicke der Holzprobe

Die Grauwertabstufung (Kontrast) der abgebildeten Probe auf dem Film ist abhängig von der Probendicke und der Strahlenqualität (kV).

7) Celluloseacetat der Firma Worbla AG, Kunststoffwerke, $\mathrm{CH}-3063$ Papiermühle - Bern.

8) Die Filme wurden mit einer Lichtmeßspalte von $1,6 \mathrm{~mm}$ Höhe und $0,06 \mathrm{~mm}$ Breite $\left(0,096 \mathrm{~mm}^{2}\right.$ Fläche) ausgemessen.

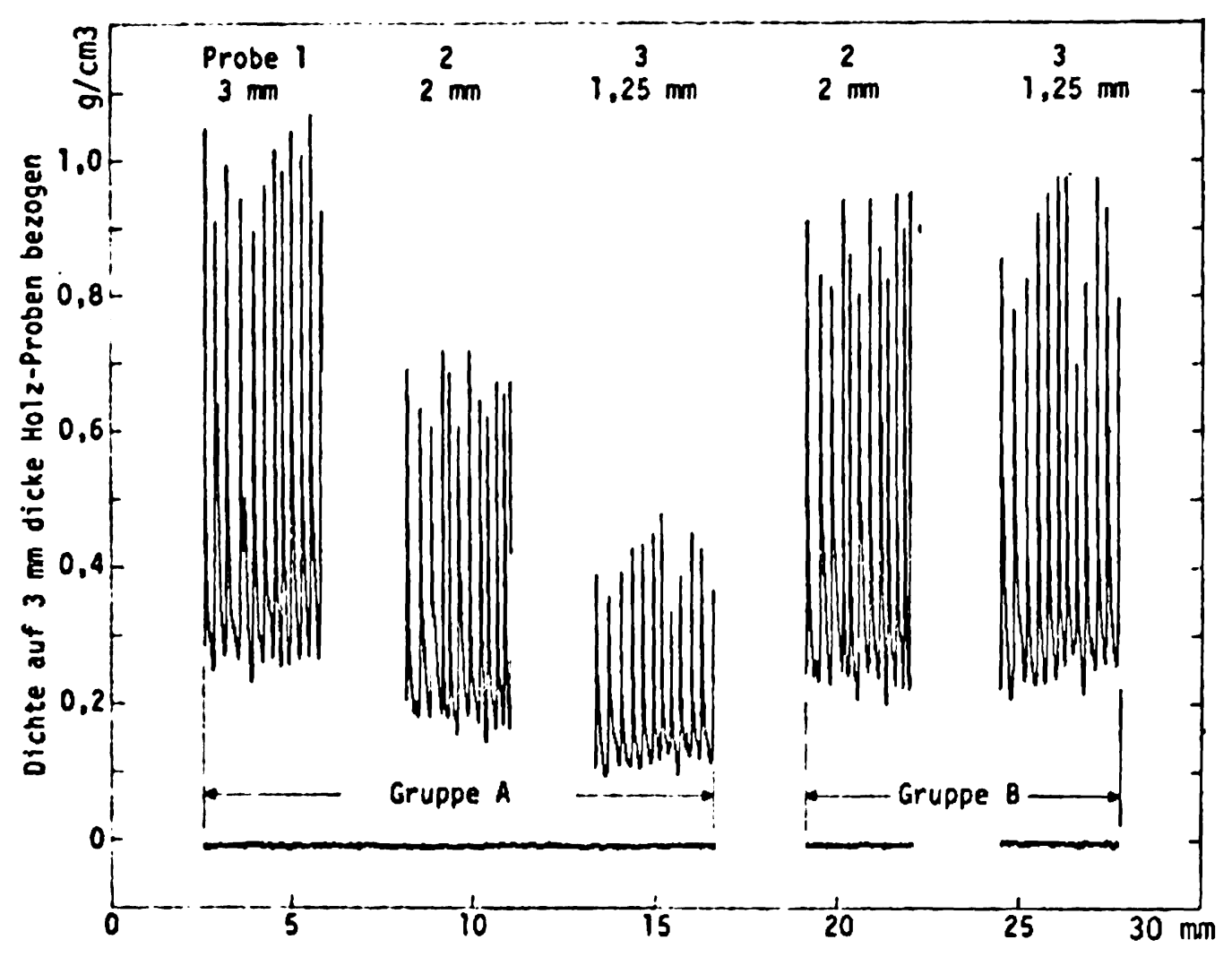

Abb. 8. Einfluß der Bestrahlungszeit und der Probendicke auf den Kontrast

Gruppe A: Fichten-Proben von $3 \mathrm{~mm}, 2 \mathrm{~mm}$ und I, $25 \mathrm{~mm}$ wurden einheitlich $6 \mathrm{~min}$. lang bestrahlt. Der Dichtekontrast sinkt mit abnehmender Probendicke. Die absoluten Dichtewerte (Skala) gelten nur für die $3 \mathrm{~mm}$-Probe.

Gruppe B: Proben von $2 \mathrm{~mm}$ und $1,25 \mathrm{~mm}$ wurden $25 \mathrm{bzw}$. $180 \mathrm{~min}$. lang bestrahlt. Mit Verlängerung der Bestrahlungszeit kann der Kontrast annähernd auf den der $3 \mathrm{~mm}$-Probe erhöht werden.

The influence of $\mathrm{X}$-ray exposure time and sample-thickness on the radiographic contrast

Group A: Spruce samples of $3 \mathrm{~mm}, 2 \mathrm{~mm}$ and $1.25 \mathrm{~mm}$ were uniformly exposed for $6 \mathrm{~min}$. The density-contrast falls with the decreasing thickness of the sample. The absolute density value (scale) is valid only for a $3 \mathrm{~mm}$ sample.

Group B: Samples of $2 \mathrm{~mm}$ and $1.25 \mathrm{~mm}$ were exposed for 25 and 180 minutes, respectively. The prolongation of exposure time can increase the contrast to approximate that which is obtained with $3 \mathrm{~mm}$ samples.

Proben mit Dicken von $3 \mathrm{~mm}$ wären zur Verarbeitung optimal, doch zwingen oft anatomische Eigenschaften des Holzes, insbesondere der schräge Faserverlauf, zur Reduktion der Probendicke bis auf I mm. Mit abnehmender Probendicke sinkt der Kontrast (Abb. 8, Gruppe A). Mit Verlängerung der Bestrahlungszeit, bei entsprechender Verminderung der Spannung, kann der Kontrast jedoch erhöht werden. Um von 3, 2 und $\mathrm{I} \mathrm{mm}$ dicken Proben ähnliche Kontraste bzw. Kurvenbilder ähnlicher Dehnung, innerhalb des optimalen Arbeitsbereiches des Mikrodensitometers, bei Verwendung eines einzigen optischen Graukeils, zu erhalten, wurden auf empirischem Wege folgende Bestrahlungsdaten für den Film Kodak RP/M ermittelt:

\begin{tabular}{c|c|c|c}
\hline $\begin{array}{c}\text { Probendicke } \\
\text { mm }\end{array}$ & $\begin{array}{c}\text { Spannung } \\
\text { kV }\end{array}$ & $\begin{array}{c}\text { Stromstärke } \\
\text { mA }\end{array}$ & $\begin{array}{c}\text { Bestrahlungszeit } \\
\text { Minuten }\end{array}$ \\
\hline 3 & I7 & 20 & 6 \\
2 & I4 & 20 & 25 \\
I,25 & I I & 20 & 180 \\
\hline
\end{tabular}

Wird ein Celluloseacetatkeil mit $15 \mathrm{kV}, 20 \mathrm{~mA}$ und 30 min. bestrahlt und der Film als Graukeil im Mikrodensitometer eingesetzt, so können verschieden dicke Proben in ähnlichem Maßstab abgebildet werden (Abb. 8).

\section{Datenerfassung}

Die gesamte Anlage besteht aus einem Mikrodensitometer, dem Steuergerät, dem Linienschreiber 
(Analogwerte) und dem Streifenlocher (Digitalwerte) (Abb. 9). Die wichtigsten Zusammenhänge zwischen den Einzelteilen sind in Abbildung ro schematisch dargestellt. Sämtliche Grauwerte, resp. Dichtewerte, des Filmes werden auf dem Linienschreiber als Kurve aufgezeichnet.

Der externe X-Y-Schreiber erlaubt die Herstellung langer Kurvenbilder ohne Unterbrechung des Arbeitsvorgangs. Die digitale Datenerfassung erfolgt über ein, von der Firma Walesch Elektronik, 8307 Effretikon ZH, Schweiz, elektronisches Datenerfassungs- und Steuergerät. Die auf der Abszisse ( $x$-Werte) registrierten Werte beziehen sich auf die Frühholz- und Spätholzbreite des Jahresringes. Auf der Ordinate ( $y$-Werte) sind die Dichteverhältnisse im Holz aufgezeichnet.

\section{I Erfassung der $x$ - Werte}

Die Schrittmotoren des Densitometers (Zusatzmotor) und des Linienschreibers sind synchronisiert. Folgende Variationen sind beim Schrittmotor des Densitometers (Film) möglich:

- Der maximale vor- und rückwärts einstellbare Vorschub beträgt $228 \mathrm{~mm}$

- Ein reduzierter Vorschub ist durch Anschläge am Mikrodensitometer, innerhalb der $228 \mathrm{~mm}$, beliebig groß wählbar.

- Die kleinste Vorschubeinheit beträgt Io Mikron ${ }^{9)}$. Bei der kontinuierlichen Registrierung wird jede Io-Mikron-Einheit auf den Linienschreiber übertragen.

${ }^{9}$ ) I Mikron $=0,001 \mathrm{~mm}$; neu: I $\mu \mathrm{m}$ (Mikrometer)
- Größere Intervalle sind von I0-990 Mikron frei wählbar und können auf den Streifenlocher übertragen werden (periodische Registrierung).

- Die Vorschubgeschwindigkeit ist frei wählbar von 4I Minuten bis 3 Minuten 38 Sekunden, pro $10 \mathrm{~cm}$ Filmlänge.

- Zur raschen Verschiebung der Filme steht ein vorund rückwärts einstellbarer Schnellauf zur Verfügung (Vorschub 53 Sek. pro ro cm Filmlänge).

Die Dehnung der Kurve in der Abszisse auf dem Linienschreiber ist im Bereich 0,02:I bis 33:I möglich.

Für die Messung der Jahrringbreiten und deren Früh- und Spätholzanteile werden die Io-Mikronschritte in Beziehung zur Dichte ( $y$-Werte) gebracht (selektive Registrierung). Die von einem Zähler ermittelten Schrittzahlen werden so lange gespeichert, bis sie einen von Hand frei eingestellten Schwellenwert überschreiten. Die Registrierung der gezählten Schritte auf dem Streifenlocher erfolgt mit einem Zusatzzeichen, das angibt, ob die Dichtekurve aufwärts (positiv) oder abwärts (negativ) überschritten worden ist.

Somit sind die Früh- und Spätholzbreiten auf einer vorbestimmten Dichtestufe (Schwellenwert) automatisch erfaßbar.

Beispiel: $\star=$ steigende Kurve $\$=$ sinkende Kurve * $0000124=$ I24 I0-Mikron-Schritte, Frühholzbreite $=1,24 \mathrm{~mm}$

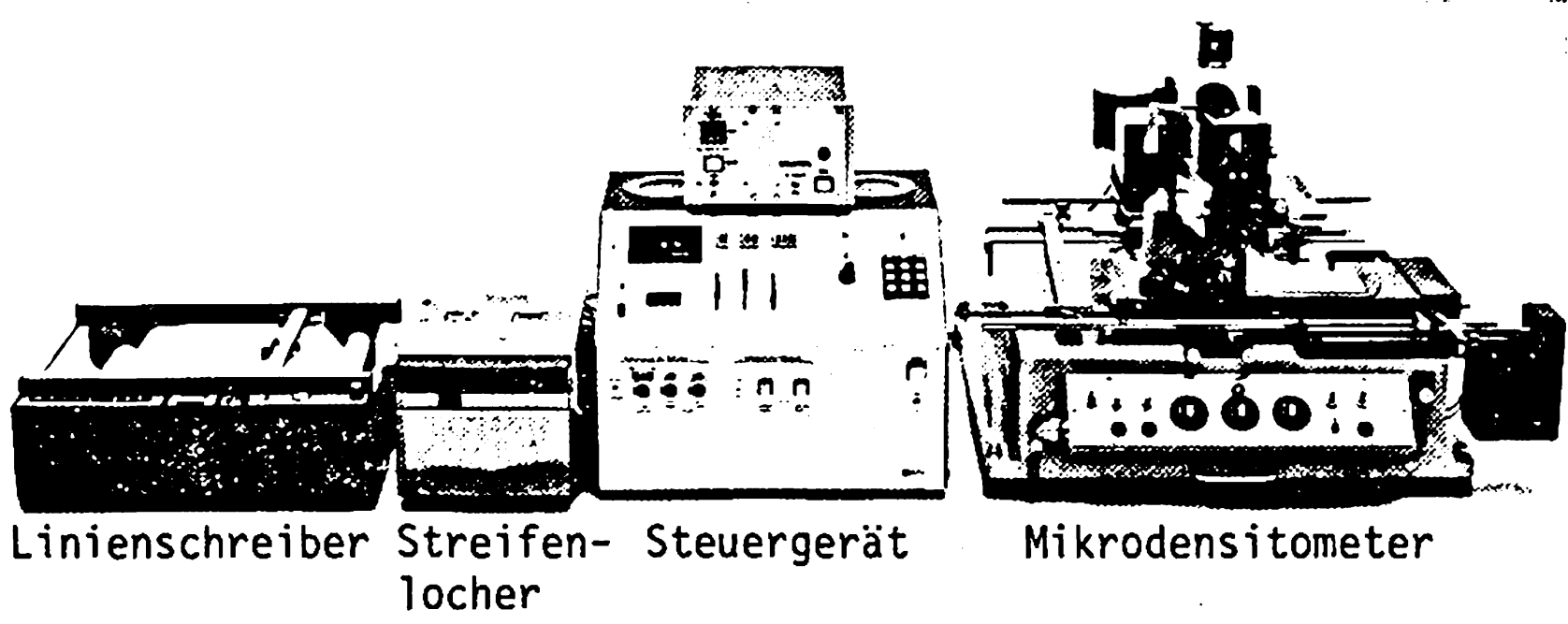

Abb. 9. Gesamtansicht der radiographischdensitometrischen Datenerfassungslage General view of the growth-ring width-density data-acquisition system

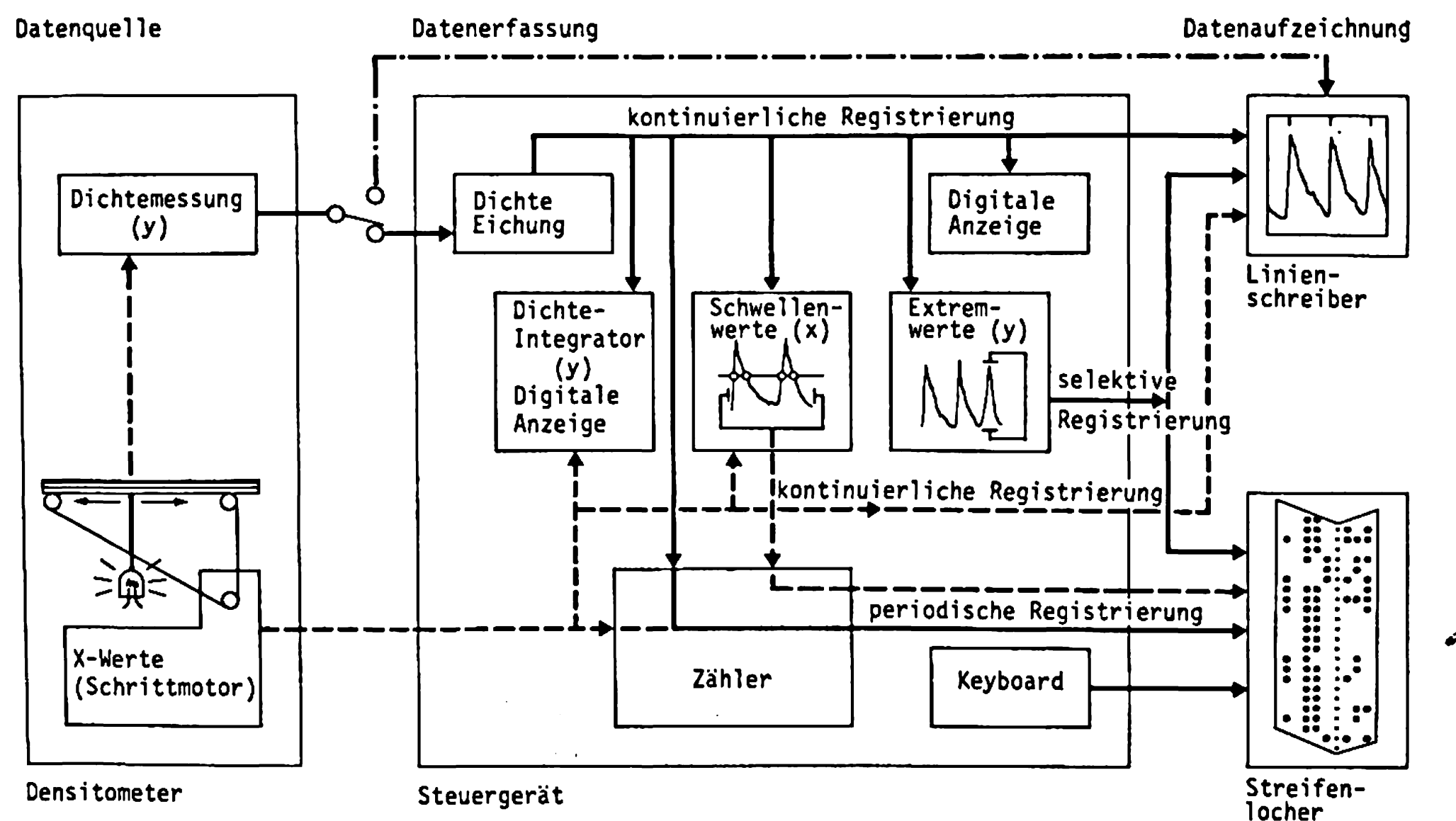

Abb. 10. Schematische Darstellung der wichtigsten Zusammenhänge innerhalb der Datenerfassungsanlage Scheme showing the principal operations of the data-acquisition system 
$\$ 000009 \mathrm{I}=9 \mathrm{I}$ 10-Mikron-Schritte, Spätholzbreite $=0,91 \mathrm{~mm}$.

\subsection{Erfassung der y-Werte}

Die vom Mikrodensitometer gemessenen Grauwertunterschiede des Films werden über ein Potentiometer und das Steuergerät auf den Linienschreiber und den Streifenlocher übertragen.

Vor der eigentlichen Dichtemessung müssen die Holzdichtekurve und die optische Dichtekurve parallel eingestellt werden (s. Abb. 7). Die Dichtewerte werden geeicht. Mit einer am Steuergerät eingebauten, digitalen Anzeige, können alle Dichtewerte zwischen einem unteren und einem oberen wählbaren Stufenwert des Eichkeils eingestellt werden. Im Anzeigefenster sind zudem während der Messung die Dichtewerte laufend ablesbar. Es werden also reelle Holzdichtewerte registriert.
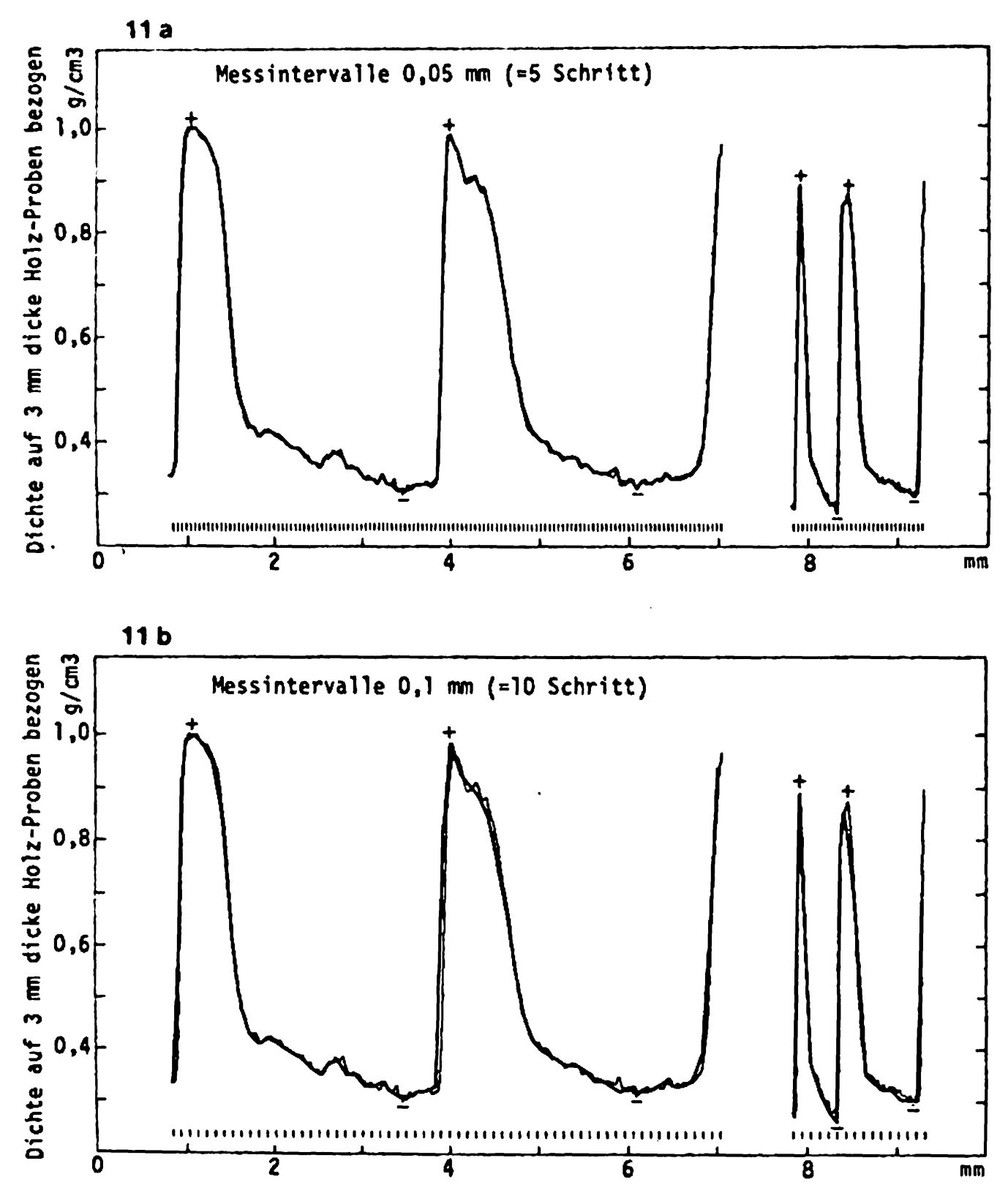

Die Registrierung der Dichten erfolgt kontinuierlich, periodisch, selektiv oder integral.

- Kontinuierliche Registrierung: Alle ro Mikron (I Schritt) wird ein Wert festgehalten.

- Periodische Registrierung: Hier werden die Dichten in regelmäßigen Abständen von ro bis 990 Mikron, bzw. I bis 99 Schritten auf dem Streifenlocher registriert. Es besteht somit die Möglichkeit, auf dem Plotter des Computers die Kurven zu reproduzieren.

Um bei der Reduktion die Datenfülle auf ein $\mathrm{Mi}$ nimum, den Kurvenverlauf nicht wesentlich, zu verändern, sind bei der periodischen Messung zusätzlich die Extremwerte (Maxima und Minima) zu registrieren.

Ohne diese zusätzlichen Werte sind 5-Schrittkurven praktisch identisch mit I-Schrittkurven (Abb. I Ia). Bei Io-Schrittkurven ergeben sich bereits Abweichungen, die im Bereiche schmaler Jahrringe falsche Maxi-
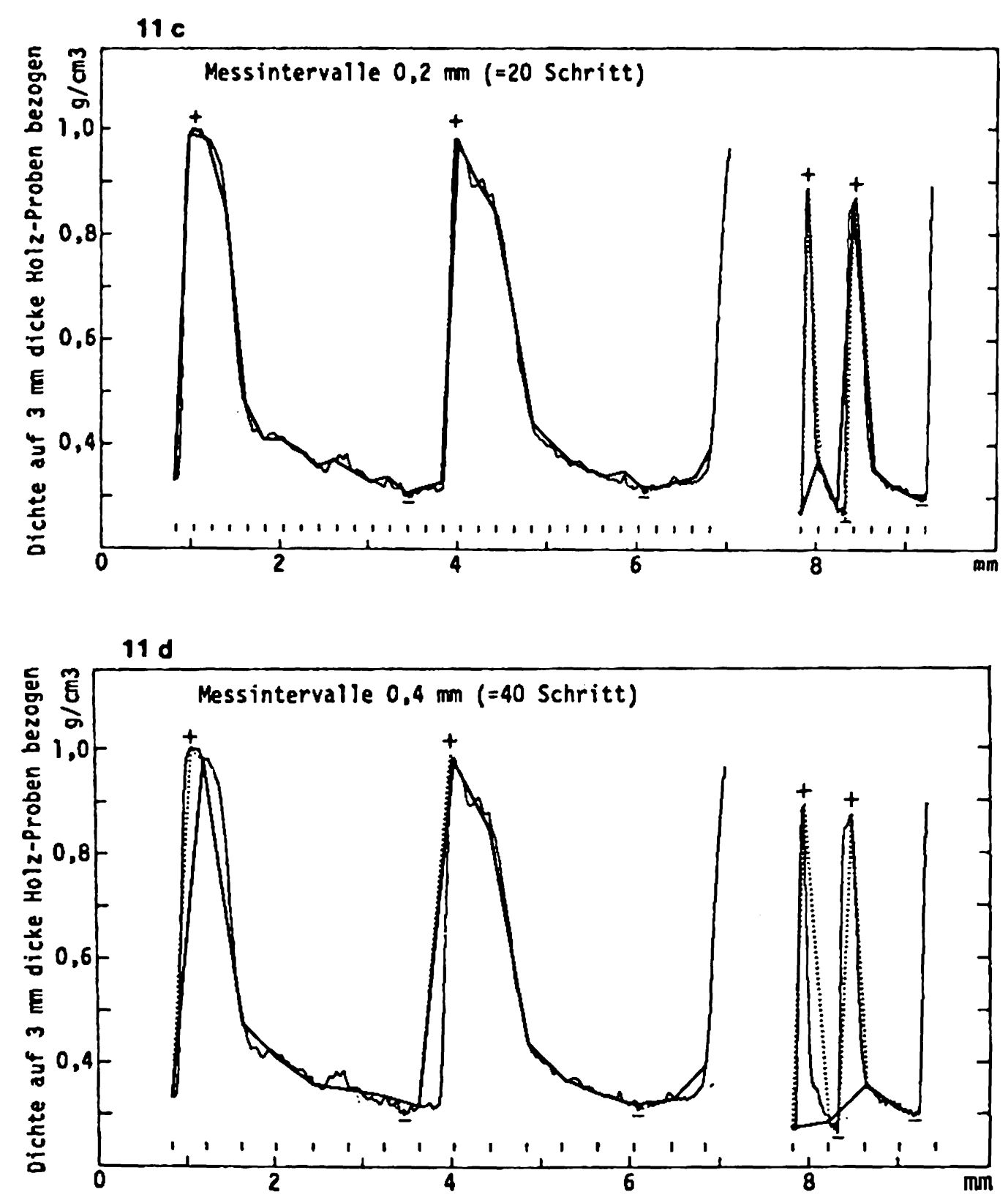

Abb. II. Aufzeichnung von Dichtekurven von breiten und schmalen Jahrringen mit maximaler und reduzierter Datenzahl (kontinuierliche, periodische und selektive Registrierung).

Kurve mit maximaler Datenzahl Kurve ohne Extremwerte

...... Kurve mit Extremwerten ( + max., - min.)

a) Bei sfacher Reduktion der Daten sind keine wesentlichen Veränderungen zu erwarten.

b) Bei Iofacher Reduktion, unter Zuzug der Extremwerte, bleibt der Kurvenverlauf im Vergleich zur optimalen Kurve gleich, Details ohne großen Belang werden eliminiert.

c) Bei zofacher Reduktion der Datenzahl ist die Kurve nur unter Zuzug der Extremwerte in der Haupttendenz abzubilden. Wesentliche Details, z. B. Doppeljahrringe, werden teilweise eliminiert.

d) Bei 4 ofacher Reduktion der Datenzahl, selbst unter Zuzug der Extremwerte, geht die Kurventendenz verloren.

The graphs of the density-curve from wide and narrow growth rings with maximum and minimum digital information (continuous, periodic, and selective recordings).

- curve with maximum digital information - continuous registration every $0.01 \mathrm{~mm}$

curve with periodic information, without the registration of the maximum $(+)$ and minimum $(\rightarrow$ ) peak values $\ldots .$. periodic curve with supplementary registration of peak values $(+\max .,-\min$.)

a) A 5 factor reduction of the digital information creates no essential change in the curve (registration of digital information

at intervals of $0.05 \mathrm{~mm}$ ).

b) After a yo factor reduction, the form of the curve remains comparable to the continuous curve if the peak values are added; only the minor details are eliminated (interval $0.1 \mathrm{~mm}$ ).

c) With a 20 factor reduction of the digital information, the form of the curve cannot be reproduced without the addition of the peak values. Essential details, i. e. double growth rings, are eliminated (interval $0.2 \mathrm{~mm}$ ).

d) With a 40 factor reduction of the digital information the curve's tendency disappears, even when the maximum and minimum peak values are added. 
malwerte zeigen (Abb. I I b). Unter Zuzug der Extremwerte ist der reelle Kurvenverlauf, mit einem Raster von 20 Schritten, noch zuverlässig nachzuzeichnen (Abb. II c). Nicht mehr tolerierbar ist ein noch geringerer Auflösungsgrad (Abb. II d).

- Selektive Registrierung: Hier werden die positiven und die negativen Scheitelwerte, (Dichtemaxima und -minima) erfaßt und auf dem StreifenLocher in digitaler Form wiedergegeben. Gleichzeitig wird auf dem Linienschreiber der Ort angegeben, wo die Werte erfaßt worden sind. Dabei unterdrückt das Steuergerät kleinere Dichteschwankungen (wählbar 10-90\% der vorangehenden Amplitude). Bei Jahrringen mit unregelmäßiger Dichteverteilung, z. B. Doppeljahrringe, werden also nur die Extremwerte erfaßt (Abb. I2a und $12 b$ ).

Mit einer Zusatzinformation an den Streifenlocher können die Daten in jahrringgerechter Form vom Streifenlesegerät ausgedruckt werden (Abb. 13).
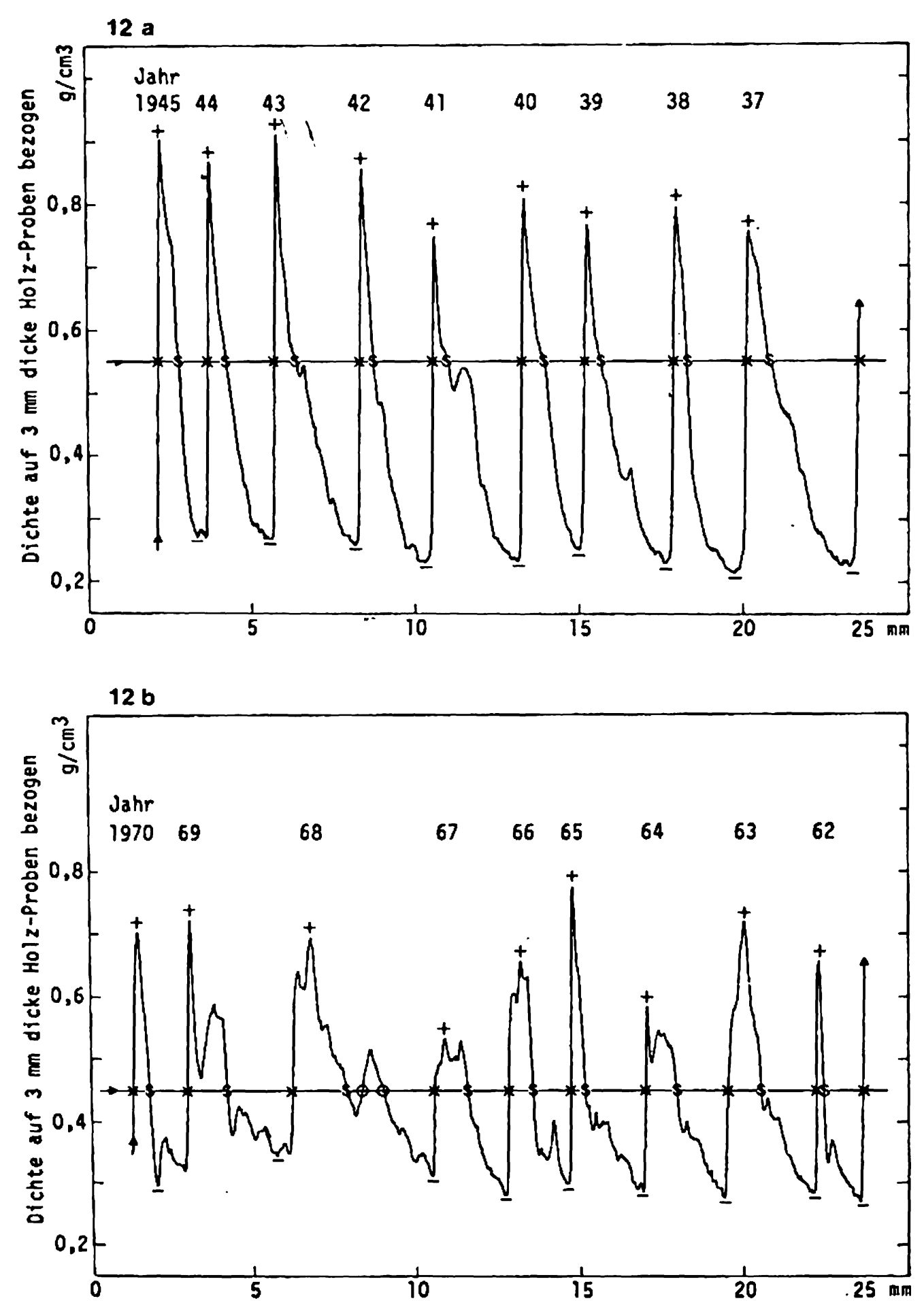

Abb. 12. Selektive Datenerfassung aus einer Kurve mit a) regelmäßigem Dichteverlauf (Abies alba)

b) unregelmäßigem Dichteverlauf (Pinus strobus)

$\star=$ positive Schwellenwertüberschneidung

$\$=$ negative Schwellenwertüberschneidung

$+=$ positiver Scheitelwert

- = negativer Scheitelwert

$0=$ Überschneidung unterdrückt (12 b : Jahr 1968)

Selected data-acquisition for a curve with

a) a uniform density progression (Abies alba)

b) non-uniform density progression (Pinus strobus)

$\star=$ value above the given density level

$\$=$ value below the given density level

$+=$ maximum positive value

$-=$ maximum negative value

0 = voluntarily non-registered value (12b : Year 1968)
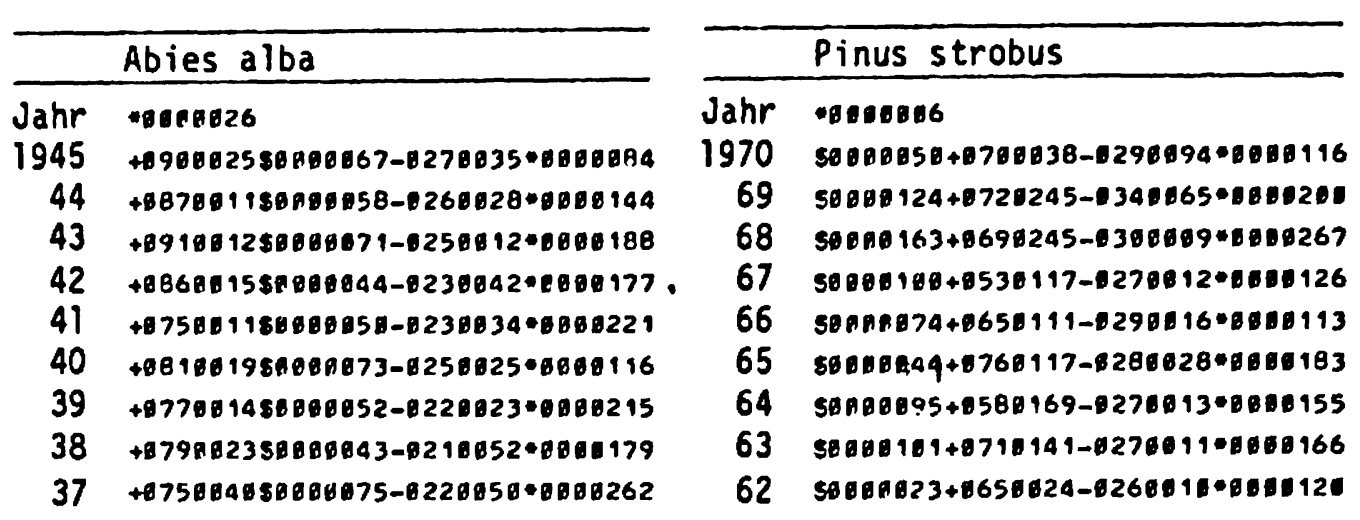

Abb. 13. Ausgewählte Daten in jahrringgerechter Form, vom Lochstreifenlesegerät ausgedruckt (Daten aus Abb. 12a und I2b)

Selective digital information grouped by growth increments by the paper reader (digital information from graphs $12 \mathrm{a}$ and $12 \mathrm{~b}$ ).

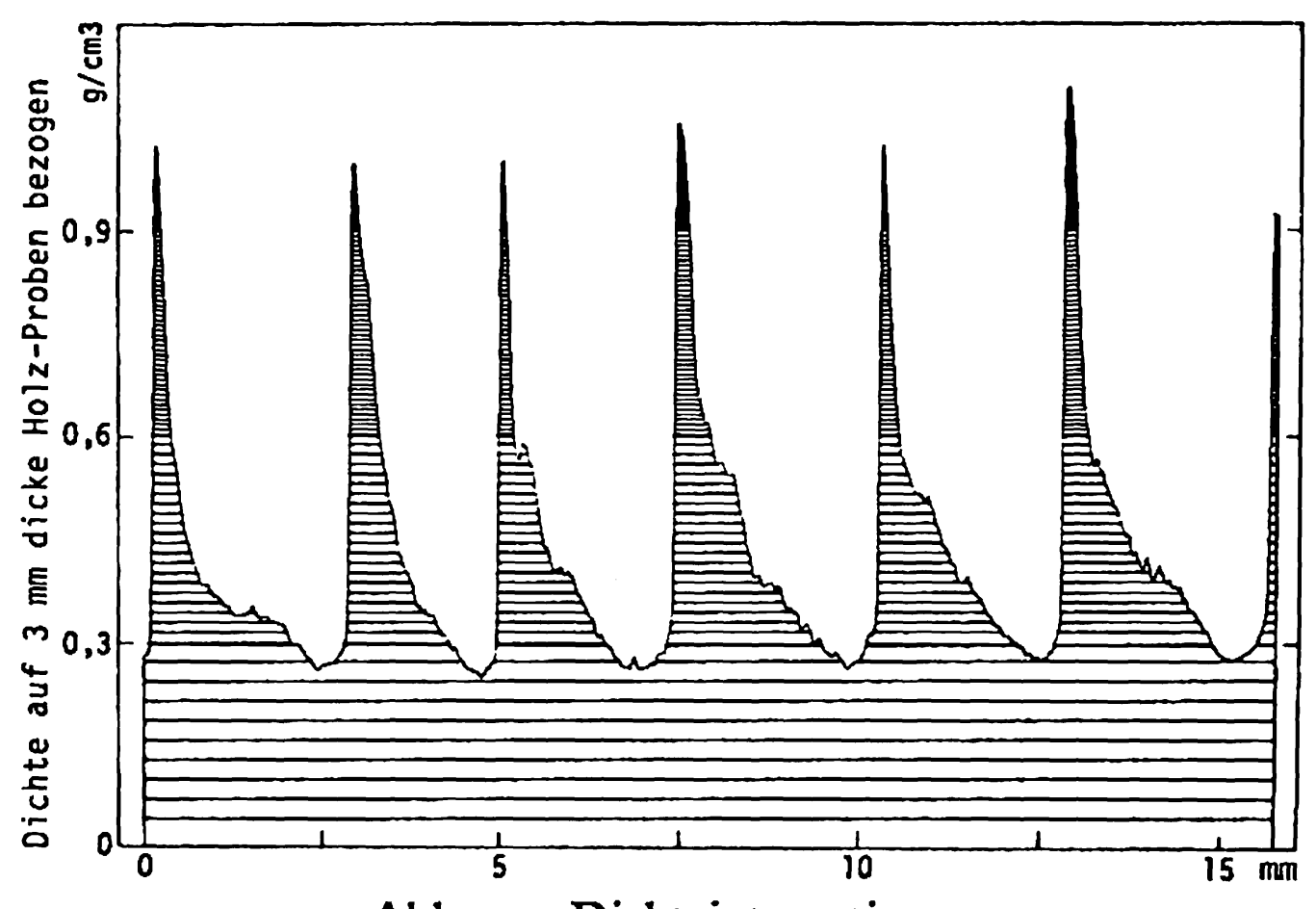

Abb. 14. Dichteintegration.

Integrierte Dichte über vorbestimmten Schwellenwerten:

Schwellenwert $\circ \mathrm{g} / \mathrm{cm}^{3}$; Dichte $0,42 \mathrm{~g} / \mathrm{cm}^{3}$

Schwellenwert $0,3 \mathrm{~g} / \mathrm{cm}^{3}$; Dichte $0,43 \mathrm{~g} / \mathrm{cm}^{3}$

Schwellenwert $0,6 \mathrm{~g} / \mathrm{cm}^{3}$; Dichte $0,82 \mathrm{~g} / \mathrm{cm}^{3}$

Schwellenwert $0,9 \mathrm{~g} / \mathrm{cm}^{3}$; Dichte $0,98 \mathrm{~g} / \mathrm{cm}^{3}$

Spätholzanteile bei den entsprechenden Schwellenwerten:

$0,3 \mathrm{~g} / \mathrm{cm}^{3}: 73 \%, 0,6 \mathrm{~g} / \mathrm{cm}^{3}: 13 \%, 0,9 \mathrm{~g} / \mathrm{cm}^{3}: 4 \%$

Density integration.

Integral density for given density levels:

$\circ \mathrm{g} / \mathrm{cm}^{3}$ given density level: $0.42 \mathrm{~g} / \mathrm{cm}^{3}$

$0.3 \mathrm{~g} / \mathrm{cm}^{3}$ given density level: $0.43 \mathrm{~g} / \mathrm{cm}^{3}$

$0.6 \mathrm{~g} / \mathrm{cm}^{3}$ given density level: $0.82 \mathrm{~g} / \mathrm{cm}^{3}$

$0.9 \mathrm{~g} / \mathrm{cm}^{3}$ given density level: $0.98 \mathrm{~g} / \mathrm{cm}^{3}$

The percentage of late wood present at a given density level: $0.3 \mathrm{~g} / \mathrm{cm}^{3}: 73 \% / 0.6 \mathrm{~g} / \mathrm{cm}^{3}: 13 \% / 0.9 \mathrm{~g} / \mathrm{cm}^{3}: 4 \%$

- Integrale Registrierung: Die durchschnittliche Holzdichte über einem vorbestimmten Schwellenwert und einer gewünschten Strecke kann mittels eines Integrators automatisch errechnet werden. Die alle Io Mikron elektronisch gemessenen Werte werden summiert und durch die Anzahl der ausgeführten Schritte dividiert. Wird der Schwellenwert auf Null eingestellt, ergibt sich die Gesamtdichte des Holzes; wird er auf einer höheren Stufe festgelegt, so ist beispielsweise die Spätholzdichte zu ermitteln (Abb. 14). Der digitale Wert ist nur optisch auf dem Fenster des Integrators abzulesen, kann aber via Keyboard auf den Streifenlocher übertragen werden.

Mit diesem Zusatzgerät, dem Integrator, ist es möglich, die radiographischen Dichtewerte zu eichen. Die Annahme gleicher Absorptionseigenschaften von $\mathrm{Holz}$ und Celluloseacetat für Röntgenstrahlen kann nun geprüft werden. Nach Bestimmung der reellen Dichte, mittels Volumen und Gewichtsbestimmung von Holz- und Celluloseacetatproben, wird die radiographische Dichte derselben Proben im Mikrodensitometer ermittelt. Hätten die beiden Materien das gleiche Absorptionsvermögen, ergäben sich mit beiden 
Methoden die gleichen Ergebnisse. Zur Ermittlung der Absorptionsraten, bzw. des Korrekturfaktors, wurden vorerst an je drei Proben von 3, 2 und 1,25 mm Dicke von Fichte, Tanne, Föhre, Lärche und Douglasie, vom gleichen Standort, die reellen und die radiographischen Dichten bestimmt. Aus der durchschnittlichen Differenz zwischen den beiden Werten ist der Korrekturfaktor zu berechnen (reelle Dichte : radiographische Dichte).

Erste Versuche zeigen, daß Holz Röntgenstrahlen um 2,5 bis II $\%$ stärker absorbiert als Celluloseacetat (Abb. 15).

Die anatomische, chemische und physikalische Heterogenität der Holzkörper äußert sich auch in den verschiedenen Absorptionsraten für Röntgenstrahlen. Folgende Tendenzen sind aus den ersten Prüfungen ablesbar:

- Holz absorbiert Röntgenstrahlen stets stärker als Celluloseacetat. Die reellen Dichten liegen deshalb tiefer als die radiographischen Dichten.

- Extrahierte Proben haben eine höhere Absorptionsfähigkeit als nicht extrahierte. Vorläufig kann für diese Erscheinung keine eindeutige Erklärung gefunden werden.

- In unseren Versuchen absorbieren nicht alle Proben Röntgenstrahlen in gleichem Maße. Es scheint, als ob die Absorptionsfähigkeit ein artspezifisches Merkmal sei. Die radiographischen Dichteerhöhungen liegen in folgenden Bereichen: Douglasie: $2,5-4,5 \%$, Lärche: $3,5-6 \%$, Waldföhre: 4-7,5\%, Tanne: 7,5-12\%, Fichte: 8-12\%.

In weiteren Versuchen muß abgeklärt werden, in welchem Maße die technische und chemische Be-

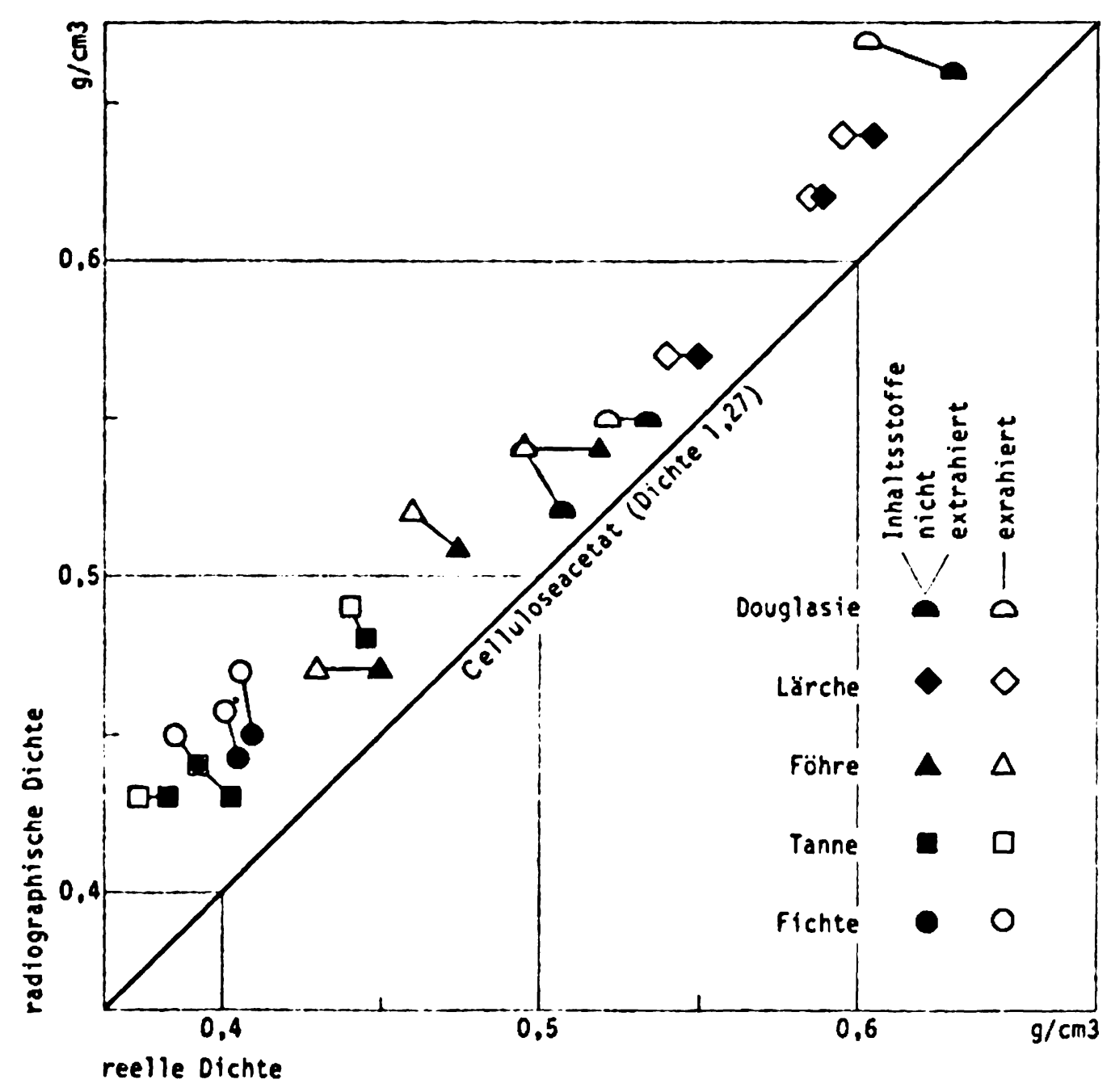

Abb. 15. Vergleich der radiographisch ermittelten Dichte (radiographische Dichte) mit der volumetrisch-gravimetrisch ermittelten Dichte (reelle Dichte)

Comparison of the density obtained by the integration of radiographic plots and the density calculated from weight and volume measures

handlung der Proben und die Standortsfaktoren die Absorptionsfähigkeit des Holzes beeinflussen.

Diese Ergebnisse zeigen, daß die reellen Dichten nicht mit einem einheitlichen Korrekturfaktor berechnet werden können. Ist dieser aber bekannt, kann die reelle Dichte mit der Formel:

$\underline{\text { Korrekturfaktor } \times \text { Dichte des Celluloseacetats } \times \text { Dicke der Celluloseacetatstufen }}$ Dicke der Holzprobe

berechnet werden.

\section{Literatur}

Arbeitsgruppe der Sektion 25 der IUFRO. 1966. Fehler bei der Zuwachsbestimmung, insbesondere bei der Zuwachsbohrung. Mitt. schweiz. Anst. forstl. Vers'wes. 42, I71-293.

Diaz-Vả $\iota, J$. E., R. Echols und W. Knigge. 1975. Vergleichende Untersuchung der Schwankungen von Tracheidendimensionen und röntgenoptisch ermittelter Rohdichte innerhalb des Jahrrings. Forstw. Cbl. 94, 161 -175 .

Fletscher, J. M. and J. F. Hughes. 1970. Uses of X-rays for density determinations and dendrochronology. Bull. Fac. for. Univ. Brit. Columbia 7, 4I-54.

Keller, R. 1968. Des caractéristiques nouvelles pour l'étude des propriétés mécaniques des bois: les composantes de la densité. Ann. sci. for. 25, 237-249.

Kusec, D. J. 1972. Twin-blades saw for precision machining of increment cores. Wood and Fiber 4, 44-49.

Lenz, O. 1957. Utilisation de la radiographie pour l'examen des couches d'accroissement. Mitt. schweiz. Anst. forstl. Vers'wes. 33, 125-134.

Lenz, O. und K. Oswald. 1971. Über Schäden durch Bohrspanentnahme an Fichte, Tanne und Buche. Mitt. schweiz. Anst. forstl. Vers'wes. 47, I-29.

Mc Neely, R., J. Neale et al. I973. Studies in dendrochronology. No. I Application of X-ray-densitometry in dendrochronology. Brock. univ. dep. geol. sci. Res. rep. ser. $16,1-30$.

Parker, M. L. and K. R. Meleskie. 1970. Preparation of Xray negatives of tree-ring specimens for dendrochronological analysis. Tree-ring Bull. 30, II-22.

Parker, M. L. 1971. Dendrochronological techniques used by the geological survey of Canada. Geol. surv. Can. Paper $71-25,1-30$.

Parker, M. L. and R. W. Kennedy. 1973. The status of radiation densitometry for measurement of wood specific gravity. Reprint from Proceed. Int. Union For. Res. Org.
(IUFRO), Div. 5 meetings Cape Town and Pretoria, SouthAfrica, Sept., Oct. I-I 7 .

Parker, M. L. and L. A. Jocza. 1973. X-ray scanning machine for tree-ring width and density analysis. Wood and Fiber 5, 192-197.

Polge, H. 1963. Une nouvelle méthode de détermination de la texture du bois: l'analyse densitométrique de clichés radiographiques. Ann. école nat. eaux et for. et de la stat. rech. et exper. 20, 53I-58I.

Polge, H. r966. Etablissement des courbes de variation de la densité du bois par exploration densitométrique de radiographies d'échantillons prélevés à la tarière sur des arbres vivants. Ann. Sci. For. 23, 1-206.

Polge, H. 1969. Vers la radiographie quantitative. Radiographie et Industrie, $1-13$.

Polge, $H$. et $F$. Thiercelin. 1970. Dégats occasionnés par les sondages à la tarière. Technique et For. 6, 629-636.

Polge, H. I97ra. Perfectionnements récents de l'analyse densitométrique du bois. Note de recherches dép. explor. et utilisation des bois. Univ. Laval 10, I-26.

Polge, H. I97 I b. Quelques conseils pratiques pour l'entretien et l'utilisation des tarières de Pressler. Technique et Forêt I 3, 25I-26r.

Polge, H. and J. W. Nicholls. 1972. Quantitative radiography and the densitometric analysis of wood. Wood Sci. 5, 5 I-59.

Reineke, L. H. 1941. A new increment core instrument and coring wrinkles. Journ. For. 39, 304-309.

Thiercelin, F. et J. R. Perrin. 1972. Débit d'échantillons d'épaisseur rigoureusement uniforme à l'aide de scies-fraises jumelées. Ann. Sci. For. 30, 295-299.

Thiercelin, F., M.-F. Arnould et al. 1972. Altérations du bois provoquées par les sondages à la tarière. Leur contrôle. Ann. Sci. For. 29, 107-1 33 . 